J. DIFFERENTIAL GEOMETRY

78 (2008) 163-191

\title{
BIMEROMORPHIC AUTOMORPHISM GROUPS OF NON-PROJECTIVE HYPERKÄHLER MANIFOLDS - A NOTE INSPIRED BY C.T. MCMULLEN
}

\author{
Keiji Oguiso
}

\begin{abstract}
Being inspired by a work of Curtis T. McMullen about a very impressive automorphism of a K3 surface of Picard number zero, we shall clarify the structure of the bimeromorphic automorphism group of a non-projective hyperkähler manifold, up to finite group factor.
\end{abstract}

\section{Introduction - Background and the statement of main result}

In his study of complex dynamics [Mc], Curtis T. McMullen has found a very impressive automorphism of a K3 surface of Picard number zero. In this note, inspired by his K3 automorphisms, we shall clarify the structure of the bimeromorphic automorphism group of a non-projective hyperkähler manifold, up to finite group factor. Our main results are Theorems (1.5), (2.4) and (3.4). Throughout this note, we work in the category of complex varieties with Euclidean topology.

For the statement of McMullen's K3 automorphism (Theorem (1.1)), we first recall a few notions from his paper $[\mathbf{M c}]$. Two complex numbers $\alpha$ and $\beta$ are multiplicatively independent if the only solution to $\alpha^{m} \beta^{n}=1$ with $(m, n) \in \mathbf{Z}^{2}$ is $(0,0)$. An automorphism $F^{*}\left(z_{1}, z_{2}\right)=\left(\alpha_{1} z_{1}, \alpha_{2} z_{2}\right)$ of the 2-dimensional unit disk $\Delta^{2}:=\left\{\left(z_{1}, z_{2}\right) \in \mathbf{C}^{2}|| z_{1}|<1,| z_{2} \mid<1\right\}$ is an irrational rotation if $\alpha_{1}$ and $\alpha_{2}$ are multiplicatively independent numbers on the unit circle $S^{1}:=\{z \in \mathbf{C}|| z \mid=1\}$. An $f$-stable domain $D \subset S$ is a Siegel disk of $f$ centered at $P$, if there are an analytic isomorphism $\varphi:(P, D) \simeq\left((0,0), \Delta^{2}\right)$ and an irrational rotation $F$ of $\Delta^{2}$ such that $f \mid D=\varphi^{-1} \circ F \circ \varphi$. As it is observed in [ibid], K3 surfaces having Siegel disk are never projective, or more strongly, of algebraic dimension 0 (cf. Example (2.5)).

McMullen has found the following:

Received 03/30/2005. 
Theorem 1.1 ([Mc]). There is a pair $(S, f)$ of a K3 surface $S$ with $\rho(S)=0$ and an automorphism $f$ of $S$ having a Siegel disk. In such a pair $(S, f)$, the topological entropy of $f$, that is, the natural logarithm of the spectral radius of $f^{*} \mid H^{2}(S, \mathbf{Z})$, is always positive. Moreover, such pairs are at most countable.

Definition 1.2. McMullen's pair is a pair $(S, f)$ in Theorem (1.1).

He constructed such a K3 automorphism starting from a certain Salem polynomial of degree 22. (See (3.1) for the definition of Salem polynomial.) Existence of McMullen's pair suggests some complexity of automorphisms of a non-projective K3 surface, and naturally leads us to the following:

Question 1.3. How complicated is the full automorphism group Aut $(S)$ of a K3 surface $S$ with Picard number $\rho(S)=0$ ?

The aim of this note is to study this question in a bit more general setting: "How does the bimeromorphic automorphism group $\operatorname{Bir}(M)$ of a non-projective hyperkähler manifold $M$ look like?" This question is posed by Y. Kawamata to me (after a preliminary version of this note).

Definition 1.4. A hyperkähler manifold is a compact complex simply-connected Kähler manifold $M$ admitting an everywhere non-degenerate global holomorphic 2-form $\sigma_{M}$ with $H^{0}\left(M, \Omega_{M}^{2}\right)=\mathbf{C} \sigma_{M}$.

Hyperkähler manifolds are even dimensional. They coincide with K3 surfaces in dimension 2 by a result of Siu $[\mathbf{S i}]$, and share many of properties with K3 surfaces [Hu]. For instance, given a hyperkähler manifold, its generic deformation is always non-projective, or more strongly, of trivial Néron-Severi group $\{0\}$ (see e.g., [Og1, Corollary 1.3]).

One of the most important properties of a hyperkähler manifold is the existence of Beauville-Bogomolov-Fujiki's form (BF-form for short) $(*, * *)$. BF-form is an integral symmetric bilinear form on $H^{2}(M, \mathbf{Z})$ of signature $\left(3,0, b_{2}(M)-3\right)$. (For more detail, see [Be1], [Fu3], an excellent survey [GHJ, Part III] by Huybrechts, and also Section 2.)

The signature (i.e., the numbers of positive-, zero-, negative-eigenvalues) of the Néron-Severi group $N S(M)$ w.r.t. BF-form is either

$$
(1,0, \rho(M)-1),(0,1, \rho(M)-1),(0,0, \rho(M)) .
$$

Here $\rho(M)$ is the Picard number of $M$. We call these three cases hyperbolic, parabolic, and elliptic respectively. Due to a very deep result by Huybrechts $[\mathbf{H u}], M$ is projective iff $N S(M)$ is hyperbolic.

Our main result is as follows:

Theorem 1.5. The bimeromorphic automorphism group $\operatorname{Bir}(M)$ of a non-projective hyperkähler manifold is an almost abelian group of rank at most $\max (\rho(M)-1,1)$. It is, in particular, finitely generated. More precisely: 
(1) If $N S(M)$ is elliptic, then $\operatorname{Bir}(M)$ falls into the exact sequence

$$
1 \longrightarrow N \longrightarrow \operatorname{Bir}(M) \longrightarrow \mathbf{Z}^{r} \longrightarrow 0
$$

where $N$ is a finite group and $r$ is either 0 or 1 . Moreover, $r=0$ if $b_{2}(M)-\rho(M)$ is odd, i.e., the rank of transcendental lattice (cf. Section 2) is odd. Moreover, in each dimension $2 m$, both $r=0$ and $r=1$ are realizable.

(2) If $N S(M)$ is parabolic, then $\operatorname{Bir}(M)$ is an almost abelian group of rank at most $\rho(M)-1$. Moreover, in dimension 2 , this estimate is optimal.

Here an almost abelian group of rank $r$ is a group isomorphic to $\mathbf{Z}^{r}$ "up to finite kernel and cokernel" (see Section 8 for the precise definition employed here and some properties we need).

As a special case of Theorem (1.5), we answer Question (1.3) fairly completely:

Corollary 1.6. Let $M$ be a K3 surface of Picard number 0 . Then Aut $(M)$ is isomorphic to either $\{i d\}$ or $\mathbf{Z}$. In particular, Aut $(M) \simeq \mathbf{Z}$ for a McMullen's K3 surface $M$. Moreover, Aut $(M) \simeq \mathbf{Z}$ for at most countably many $M$; in other words, mostly Aut $(M)=\{i d\}$.

So, the automorphism group of a McMullen's K3 surface turns out to be quite simple as a group. It will be also very interesting to view these results from the view of topological entropy, especially in connection with recent important results of Dinh and Sibony [DS1, DS2, DS3]. We also note that there is a projective hyperkähler manifold $M$ whose $\operatorname{Bir}(M)$ is not almost abelian (cf. [Og2, Og3]).

As it is well known, there are hyperkähler manifolds of dimension $2 m$ for each $m \geq 2$; the Douady space (Hilbert scheme) $S^{[m]}$ of 0 dimensional analytic subspaces $\left(Z, \mathcal{O}_{Z}\right)$ of length $m$ on a K3 surface $S$, and manifolds $D_{m}$ obtained by a small deformation of $S^{[m]}([\mathbf{B e} \mathbf{1}])$. We have $b_{2}\left(D_{m}\right)=b_{2}\left(S^{[m]}\right)=23$ by [ibid]. Moreover, $\rho\left(D_{m}\right)=0$ and $b_{2}\left(D_{m}\right)-\rho\left(D_{m}\right)=23$, an odd number, for a sufficiently generic $D_{m}$ in the Kuranishi family (see e.g., [Og1]). As an immediate consequence of Theorem (1.5)(1), one has:

Corollary 1.7. Bir $\left(D_{m}\right)$ is a finite group for $D_{m}$ with $\rho\left(D_{m}\right)=0$.

Compare Corollary (1.7) with (1.6).

To conclude this introduction, it is also worth mentioning that McMullen's pair can be used, as in Voisin's paper [Vo], to produce a 4-dimensional simply-connected counterexample of Kodaira's problem (1.8 below): 
Problem 1.8 (cf. $[\mathbf{C P}]$ ). Is any compact Kähler manifold algebraically approximated? Or more precisely, for a given compact Kähler manifold $X$, is there a small deformation $\pi: \mathcal{X} \longrightarrow \mathcal{B}$ such that $\mathcal{X}_{0} \simeq X$ and $\mathcal{X}_{t_{\nu}}$ is projective for a sequence $\left(t_{\nu}\right)$ converging to 0 ?

Section 2 is a preliminary section, which is based on [GHJ, Part III], about some basic properties of hyperkähler manifolds and their bimeromorphic automorphisms. Following $[\mathbf{M c}]$, we clarify a close relation between bimeromorphic automorphism in the elliptic $N S(M)$ case and Salem polynomials in Section 3. We prove the main part of Theorem (1.5)(1) in Section 4 and the main part of Theorem (1.5)(2) in Section 5. In Sections 6 and 7, we show Corollary (1.6), the last statements in Theorem (1.5)(1) and (2). Section 8 is a sort of appendix about basic properties of almost abelian groups, which are used thoughout this note.

Acknowledgement. I would like to express my deep thanks to Professors A. Fujiki, D. Huybrechts, Y. Kawamata, and J.H. Keum for their very valuable discussions and comments. I would also like to express my thanks to Professors T.-C. Dinh, John H. Coates, I. Enoki, R. Goto, K. Ohno, T. Peternell for their interest in this work. The final version has been completed during my stay at KIAS in March 2005. I would like to express my thanks to Professors J. Hwang and B. Kim for invitation.

\section{Some basic properties of Hyperkähler manifolds}

In this section, we first recall the definition and some basics about Beauville-Bogomolov-Fujiki's form (BF-form) and bimeromorphic automorphisms from $[\mathbf{B e 1}],[\mathbf{F u}]$ and [GHJ, Part III]. We then study various representations of the bimeromorphic automorphism group of a hyperkähler manifold. Theorem (2.4) is new.

1. Throughout Section 2 , let $M$ be a hyperkähler manifold of complex dimension $2 m$ with $H^{0}\left(M, \Omega_{M}^{2}\right)=\mathbf{C} \sigma_{M}$. We may normailize $\sigma_{M}$ as $\int_{M}\left(\sigma_{M} \bar{\sigma}_{M}\right)^{m}=1$. We note that $H^{2}(M, \mathbf{Z})$ is torsion free, because $\pi_{1}(M)=\{1\}$. Define a (real valued) quadratic form $\tilde{q}_{M}$ on $H^{2}(M, \mathbf{Z})$ by

$$
\begin{aligned}
\tilde{q}_{M}(\alpha):=\frac{m}{2} \int_{M} \alpha^{2}\left(\sigma_{M} \bar{\sigma}_{M}\right)^{m-1} & \\
& +(1-m)\left(\int_{M} \alpha \sigma_{M}^{m-1} \bar{\sigma}_{M}^{m}\right) \cdot\left(\int_{M} \alpha \sigma_{M}^{m} \bar{\sigma}_{M}^{m-1}\right) .
\end{aligned}
$$

By $[\mathbf{B e 1}],[\mathbf{F u} 3]$, there is a unique positive constant $c_{M}>0$ such that the symmetric bilinear form

$$
(*, * *): H^{2}(M, \mathbf{Z}) \times H^{2}(M, \mathbf{Z}) \longrightarrow \mathbf{Z},
$$


associated with $q_{M}:=c_{M} \tilde{q}_{M}$, is integral and primitive, in the sense that $(*, * *) / k\left(k \in \mathbf{Z}_{>0}\right)$ is integral valued iff $k=1$. Beauville-BogomolovFujiki's form, BF-form for short, is this bilinear form $(*, * *)$. BF-form $(*, * *)$ coincides with the cup product when $\operatorname{dim} M=2$, i.e., when $M$ is a $\mathrm{K} 3$ surface, and $(*, * *)$ enjoys similar properties to the cup product of the K3 surface.

2. We always regard $H^{2}(M, \mathbf{Z})$ as a lattice by BF-form. Then $H^{2}(M, \mathbf{Z})$ is of signature $\left(3,0, b_{2}(M)-3\right)$, i.e., the numbers of positive-, zero- and negative-eigenvalues are $3,0, b_{2}(M)-3$. More precisely, by [Be1] one has that:

$$
\begin{gathered}
\left(\sigma_{M}, \sigma_{M}\right)=0,\left(\sigma_{M}, \bar{\sigma}_{M}\right)>0, \\
\left(\sigma_{M}, H^{1,1}(M, \mathbf{R})\right)=\left(\bar{\sigma}_{M}, H^{1,1}(M, \mathbf{R})\right)=0,
\end{gathered}
$$

and that if $\eta \in H^{1,1}(M, \mathbf{R})$ is a Kähler class, then $(\eta, \eta)>0$ and $(*, * *)$ is negative definite on the orthogonal complement of $\mathbf{R}\langle\eta\rangle$ in $H^{1,1}(M, \mathbf{R})$. Thus the BF-form on $H^{1,1}(M, \mathbf{R})$ is of signature $\left(1,0, h^{1,1}(M)-1\right)$, and $\left\{x \in H^{1,1}(M, \mathbf{R}) \mid\left(x^{2}\right)>0\right\}$ has two connected components. We define the positive cone $\mathcal{C}=\mathcal{C}(M)$ to be the component which contains the Kähler cone $\mathcal{K}(M)$, i.e., the (open convex) cone of $H^{1,1}(M, \mathbf{R})$ consisting of Kähler classes of $M$.

3. Let $N S(M)$ be the Néron-Severi group of $M$, i.e., the subgroup of $H^{2}(M, \mathbf{Z})$ generated by the first Chern classes of holomorphic line bundles. We regard $N S(M)$ as a (possibly degenerate) sublattice of $H^{2}(M, \mathbf{Z})$ by $(*, * *)$. The transcendental lattice $T(M)$ of $M$ is the pair of the minimum subgroup $T$ of $H^{2}(M, \mathbf{Z})$ such that $\mathbf{C} \sigma_{M} \in T \otimes \mathbf{C}$ and $H^{2}(M, \mathbf{Z}) / T$ is torsion-free, and the bilinear form $(*, * *)_{T \times T}$. By the Lefschetz (1,1)-theorem and non-degeneracy of the BF-form on $H^{2}(M, \mathbf{Z})$, we have that

$$
\begin{aligned}
N S(M) & =H^{2}(M, \mathbf{Z}) \cap H^{1,1}(M), \\
T(M) & =\left\{x \in H^{2}(M, \mathbf{Z}) \mid(x, N S(M))=0\right\} .
\end{aligned}
$$

Note that $T(M) \otimes \mathbf{R}$ contains a positive 2 -plane $P:=\mathbf{R}\left\langle\operatorname{Re} \sigma_{M}, \operatorname{Im} \sigma_{M}\right\rangle$. Then, as in the K3 case [Ni], the signature of $N S(M)$ is any one of

$$
(1,0, \rho(M)-1),(0,1, \rho(M)-1),(0,0, \rho(M)) .
$$

We call these three cases hyperbolic, parabolic, elliptic, respectively. By a fundamental result of Huybrechts $[\mathbf{H u}]$ (see also [GHJ, Proposition 26.13]), $M$ is projective iff $N S(M)$ is hyperbolic.

In the hyperbolic and elliptic cases, one has also

$$
N S(M) \cap T(M)=\{0\} \text { and }\left[H^{2}(M, \mathbf{Z}): N S(M) \oplus T(M)\right]<\infty .
$$

In the parabolic case, $N S(M) \cap T(M)=\mathbf{Z} v$ with $\left(v^{2}\right)=0$, and $N S(M)+T(M)$ is of co-rank one in $H^{2}(M, \mathbf{Z})$. 
4. Let $g \in \operatorname{Bir}(M)$ be a bimeromorphic automorphism of $M$. We choose a Hironaka's resolution $([\mathbf{H i}]) \pi_{1}: Z \longrightarrow M$ of the indeterminacy $I(g)$ of $g$ (of the source $M$ ) and denote by $\pi_{2}: Z \longrightarrow M$ the induced morphism to the target $M$. Recall that $I(g)$ is the minimum (necessarily Zariski closed) subset of $M$ such that $g$ is holomorphic over $U:=U(g):=$ $M \backslash I(g)$, and that codim $I(g) \geq 2$. Let $\left\{E_{j}\right\}_{j \in J}$ be the set of exceptional prime divisors of $\pi_{1}$. Put $E:=\cup_{j \in J} E_{j}$ and $I^{\prime}(g):=\pi_{2}(E)$. These (with reduced structure) are closed analytic subsets of $Z$ and (the target) $M$ respectively. Since $\pi_{1} \mid Z \backslash E: Z \backslash E \simeq M \backslash I(g)$ is an isomorphism, we have $g(U)=M \backslash I^{\prime}(g)$. In particular, $g(U)$ is a Zariski open subset of the target $M$.

Since $\pi_{1}$ is a successive blow-up in smooth centers, one has:

$$
H^{2}(Z, \mathbf{K})=\pi_{1}^{*} H^{2}(M, \mathbf{K}) \oplus \oplus_{j \in J} \mathbf{K}\left[E_{j}\right] .
$$

Here $\mathbf{K}$ is any ring containing $\mathbf{Z}$ (mostly, $\mathbf{Z}, \mathbf{Q}, \mathbf{R}$, or $\mathbf{C}$ ).

Recall that $K_{M}=0$. Then, as divisors, $K_{Z}=\sum_{j \in J} a_{j} E_{j}$ for some positive integers $a_{j}>0$ and $\left(\pi_{2}\right)_{*} K_{Z}=K_{M}=0$. Since $M$ is Kähler, this implies that the analytic subset $I^{\prime}(g)=M \backslash g(U)$ is of codimension $\geq 2$ as well.

Let $\iota: U \longrightarrow M$ be the natural inclusion. Recall that $\operatorname{codim} M \backslash U \geq 2$ and $\Omega_{M}^{2}$ is locally free. Then one has $\iota_{*} H^{2}\left(U, \Omega_{U}^{2}\right)=H^{2}\left(M, \Omega_{M}^{2}\right)=$ $\mathbf{C} \sigma_{M}$. Thus,

$$
g^{*} \sigma_{M}:=\left(\iota_{U}\right)_{*}(g \mid g(U))^{*}\left(\sigma_{M} \mid g(U)\right)
$$

is a holomorphic 2-form on $M$. Hence $g^{*} \sigma_{M}$ is a non-zero multiple of $\sigma_{M}$, and is in particular everywhere non-degenerate. So, the morphism $g \mid U$ : $U \longrightarrow g(U)$ is an isomorphism and we have then that $I\left(g^{-1}\right)=I^{\prime}(g)$ as well (cf. [GHJ, Proposition 21.6]). Since $\pi_{i}^{*} H^{0}\left(M, \Omega_{M}^{2}\right)=H^{0}\left(Z, \Omega_{Z}^{2}\right)$, we also have $\pi_{1}^{*}\left(g^{*} \sigma_{M}\right)=\pi_{2}^{*} \sigma_{M}$ as forms. Thus $g^{*} \sigma_{M}=\left(\pi_{1}\right)_{*} \pi_{2}^{*} \sigma_{M}$ in $H^{2}(M, \mathbf{C})$. In particular, the definition of $g^{*}$ here is compatible with $g^{*}$ on $H^{2}(M, \mathbf{Z})$ defined below.

5. Let us study representations of $\operatorname{Bir}(M)$.

Lemma 2.1. Let $g \in \operatorname{Bir}(M)$ and define $\chi(g) \in \mathbf{C}$ by $g^{*} \sigma_{M}=$ $\chi(g) \sigma_{M}$. Then $\chi(g)$ is on the unit circle $S^{1}$, i.e., $|\chi(g)|=1$.

Proof. Set $\sigma^{\prime}=g^{*} \sigma_{M}$. Then, by $\pi_{1}^{*} \sigma^{\prime}=\pi_{2}^{*} \sigma_{M}$,

$$
\begin{aligned}
\int_{M}\left(\sigma^{\prime} \overline{\sigma^{\prime}}\right)^{m} & =\int_{Z}\left(\pi_{1}^{*} \sigma^{\prime} \overline{\pi_{1}^{*} \sigma^{\prime}}\right)^{m} \\
& =\int_{Z}\left(\pi_{2}^{*} \sigma_{M} \overline{\pi_{2}^{*} \sigma_{M}}\right)^{m}=\int_{M}\left(\sigma_{M} \overline{\sigma_{M}}\right)^{m}=1 .
\end{aligned}
$$

Since $\sigma^{\prime}=\chi(g) \sigma_{M}$, this impies the result.

q.e.d.

The correspondence $Z$ also induces a group automorphism $g^{*}=$ $\left(\pi_{1}\right)_{*} \pi_{2}^{*}$ of $H^{2}(M, \mathbf{Z})$ (and a $\mathbf{K}$-linear automorphism on $H^{2}(M, \mathbf{K})$ as well). By the observation above, $g^{*}$ preserves the Hodge decomposition. 
Note that if $g^{*} \eta=\eta^{\prime}$, then $\pi_{1}^{*} \eta^{\prime}=\pi_{2}^{*} \eta+\sum_{j \in J} a_{j} E_{j}$ in $H^{2}(M, \mathbf{K})$ for some $a_{j} \in \mathbf{K}$. It is observed by [GHJ, Section 27] that $\left(\pi_{1}^{*} \sigma_{M}\right)^{m-1} \mid E_{i}=$ 0 for each exceptional prime divisor $E_{i}$. Now, by calculating $q_{M}\left(g^{*} \eta\right)$ and $q_{M}(\eta)$ (by pulling back to $Z$ as above), one obtains:

Theorem 2.2 ([GHJ, Proposition 25.14]). $\operatorname{Bir}(M)$ acts on $H^{2}(M, \mathbf{Z})$ as a Hodge isometry with respect to BF-form. Moreover, $\operatorname{Bir}(M)$ preserves the positive cone $\mathcal{C}$.

Proof. Since the last claim is implicit there, we shall give its proof here. Let $\eta$ be a Kähler class and put $\eta^{\prime}=g^{*} \eta$. It suffices to show that $\left(\eta^{\prime}, \eta\right) \geq 0$. We can write $\pi_{1}^{*} \eta^{\prime}=\pi_{2}^{*} \eta+\sum_{j \in J} a_{j} E_{j}$ with $a_{j} \in \mathbf{R}$. By using the definition of BF-form and the fact that $\left(\pi_{1}^{*} \sigma_{M}\right)^{m-1} \mid E_{i}=0$, one can calculate

$$
\begin{aligned}
\left(\eta^{\prime}, \eta\right)=c \int_{M}\left(g^{*} \eta\right) \eta(\sigma \bar{\sigma})^{m-1} & =c \int_{Z}\left(\pi_{1}^{*} \eta^{\prime}\right)\left(\pi_{1}^{*} \eta\right) \pi_{1}^{*}(\sigma \bar{\sigma})^{m-1} \\
& =c \int_{Z}\left(\pi_{2}^{*} \eta\right)\left(\pi_{1}^{*} \eta\right) \pi_{1}^{*}(\sigma \bar{\sigma})^{m-1} \geq 0 .
\end{aligned}
$$

Here $c$ is a positive constant. The last inequality is because each of three terms (in the last integral) is a pull back of a weakly-positive form by a morphism.

q.e.d.

6. We have then four natural representations of $\operatorname{Bir}(M)$ :

$$
\begin{aligned}
r & : \operatorname{Bir}(M) \longrightarrow \mathrm{O}\left(H^{2}(M, \mathbf{Z})\right) ; f \mapsto f^{*}, \\
\chi & : \operatorname{Bir}(M) \longrightarrow \mathbf{C}^{\times} \text {by } f^{*} \sigma_{M}=\chi(f) \sigma_{M}, \\
r_{T} & : \operatorname{Bir}(M) \longrightarrow \mathrm{O}(T(M)) ; f \mapsto f^{*} \mid T(M), \\
r_{N S}: \operatorname{Bir}(M) & \longrightarrow \mathrm{O}(N S(M)) ; f \mapsto f^{*} \mid N S(M) .
\end{aligned}
$$

The next important theorem due to Huybrechts $[\mathbf{H u}$, Section 9] reduces our study of $\operatorname{Bir}(M)$ to its representation $r(\operatorname{Bir}(M))$ :

Theorem $2.3([\mathbf{H u}])$. $\left|\operatorname{Ker}\left(r: \operatorname{Bir}(M) \longrightarrow \mathrm{O}\left(H^{2}(M, \mathbf{Z})\right)\right)\right|<\infty$.

Proof. This is so important for us that we shall review the proof here. Let $K$ be the kernel. Let $g \in K$. Then $g^{*}(\eta)=\eta$ for a Kähler class $\eta$. Thus $g \in \operatorname{Aut}(M)$ by a result of Fujiki [Fu1] (see also [GHJ, Proposition 27.7] for a useful refinement). Recall that by Yau's solution of Calabi's conjecture [Ya1, Ya2], there is a unique Ricci-flat $\tilde{\eta}$ metric associated to the class $[\eta]$. Thus, we also have $K<\mathrm{O}(M, \tilde{\eta})$, the group of isometries of $(M, \tilde{\eta})$. This $\mathrm{O}(M, \tilde{\eta})$ is a compact subgroup of $\operatorname{Diff}(M)$ (with respect to the compact-open topology), because $M$ is compact (see for instance $[\mathbf{H e}]$ or $[\mathbf{K b}])$. On the other hand, Aut $(M)$ forms a closed discrete subgroup of $\operatorname{Diff}(M)$ by $H^{0}\left(M, T_{M}\right)=0$. Thus, $\mid \operatorname{Aut}(M) \cap$ $\mathrm{O}(M, \tilde{\eta}) \mid<\infty$ and hence $|K|<\infty$ as well. 
The next theorem slightly strengthens earlier results of Nikulin [Ni] and Beauville [Be2, Proposition 7]:

Theorem 2.4. Let $M$ be a hyperkähler manifold. Let $G<\operatorname{Bir}(M)$. Set $A^{\prime}:=\chi(G)$ and $A:=r_{T}(G)$. Then:

(1) The characteristic polynomial $\Phi(x)$ of $g^{*} \mid T(M)(g \in G)$ is primary in $\mathbf{Z}[x]$, i.e., $\Phi(x)$ is of the form $f(x)^{m}$ for an irreducible polynomial $f(x) \in \mathbf{Z}[x]$.

(2) The natural homomorphism

$$
\psi: A \longrightarrow A^{\prime} ; g^{*} \mid T(M) \mapsto \chi(g)
$$

is an isomorphism. In particular, $A$ is an abelian group.

(3) $A^{\prime}<S^{1}$.

(4) If $N S(M)$ is hyperbolic, then $A \simeq A^{\prime}$ is a finite cyclic group $\mu_{n}$, where $n \geq 1$ is some integer s.t. $\varphi(n) \mid \operatorname{rank} T(M)$. Here $\varphi(n):=$ $\left|(\mathbf{Z} / n \mathbf{Z})^{\times}\right|$, i.e., the cardinality of the inverible elements of the ring $\mathbf{Z} / n \mathbf{Z}$.

(5) If $N S(M)$ is parabolic, then $A \simeq A^{\prime}=\{1\}$.

(6) If $N S(M)$ is elliptic, then $A \simeq A^{\prime}$ is torsion free, i.e., either $A^{\prime}=\{1\}$ or ord $(\chi(g))=\infty$ for all $g \in G \backslash\{1\}$. Moreover, $\operatorname{Ker}\left(\chi: G \longrightarrow A^{\prime}\right)$ is finite, and $|G|=\infty$ iff $\left|A^{\prime}\right|=\infty$. Here ord $(a)$ is the order of an element $a$ in a group.

Proof. Let us show (1). If otherwise, we have a decomposition in $\mathbf{Z}[x]$ :

$$
\Phi(x)=f_{1}(x) f_{2}(x) \text { s.t. }\left(f_{1}(x), f_{2}(x)\right)=1 .
$$

This gives a decomposition of $T(M)_{\mathbf{Q}}$, say $T(M)_{\mathbf{Q}}=V_{1} \oplus V_{2}$, such that both $V_{i}$ are $g^{*}$-stable and the characteristic polynomial of $g^{*} \mid V_{i}$ is $f_{i}(x)$ for each $i=1,2$. Since $f_{1}$ and $f_{2}$ have no common zero, $\sigma_{M}$ then belongs to one of $V_{i} \otimes \mathbf{C}$, a contradiction to the minimality of $T(M)$.

Let us show (2). Clearly $\psi$ is surjective. Take $g \in G$ such that $\chi(g)=1$, i.e., $g^{*} \sigma_{M}=\sigma_{M}$. Let $V:=T(M)^{g^{*}}$ be the set of $g$-invariant elements of $T(M)$. Then $\sigma_{M} \in V \otimes \mathbf{C}$, whence, by the minimality of $T(M)$, we have $V=T(M)$, i.e., $g^{*} \mid T(M)=i d$. The last statement of (2) is now clear, because so is $A^{\prime}<\mathbf{C}^{\times}$. The assertion (3) is proved in Lemma (2.1).

Let us show (4). We first show that $\chi(g)$ is a root of unity for $g \in G$. Set

$$
N:=P_{T(M) \otimes \mathbf{R}}^{\perp}:=\{v \in T(M) \otimes \mathbf{R} \mid(v, P)=0\} .
$$

Here $P=\mathbf{R}\left\langle\operatorname{Re} \sigma_{M}, \operatorname{Im} \sigma_{M}\right\rangle$ is the positive 2-plane. Then $T(M) \otimes \mathbf{R}=$ $P \oplus N$ and $N$ is negative definite. Put $g_{T}:=g^{*} \mid T(M)$. Then $g_{T}(P)=P$ and $g_{T}(N)=N$. Hence $g_{T} \in \mathrm{O}(P) \times \mathrm{O}(N)$. Thus the eigenvalues of $g_{T}$ are on the unit circle. On the other hand, the eigenvalues of $g_{T}$ are all algebraic integers, because $g_{T} \in \mathrm{O}(T(M))$ so that $g_{T}$ is represented by an integral matrix. Hence, they are roots of unity by Kronecker's 
theorem (see e.g., [Ta, Section 9.2]). Since $\chi(g)$ is one of the eigenvalue of $g_{T}$, it is also a root of unity, say, a primitive $n(g)$-th root of unity. By (1), $\varphi(n(g)) \mid \operatorname{rank} T(M)$. Hence, the orders $n(g)$ of $g(g \in G)$ are bounded. Thus $A^{\prime} \simeq \mu_{n}$ for some $n$ with $\varphi(n) \mid \operatorname{rank} T(M)$.

Let us show (5). Set $N S(M) \cap T(M)=\mathbf{Z} v$ (as before). Replacing $v$ by $-v$ if necessary, we may assume that $v \in \partial \mathcal{C}$. Then, by $g^{*}(\mathcal{C})=\mathcal{C}$, we have $g^{*}(v)=v$ for each $g \in G$. Thus $g^{*} \sigma_{M}=\sigma_{M}$ for each $g \in G$ by (1), i.e., $A^{\prime}=\{1\}$.

Finally, we shall show (6). Since $N S(M) \oplus T(M)$ is of finite index in $H^{2}(M, \mathbf{Z})$, the group $\operatorname{Ker} r_{T} / \operatorname{Ker} r$ is embedded into $\mathrm{O}(N S(M))$. Thus Ker $\chi=\operatorname{Ker} r_{T}$ is finite by $|\mathrm{O}(N S(M))|<\infty$ and by Theorem (2.2). Thus $|G|<\infty$ iff $\left|A^{\prime}\right|<\infty$. It remain to show that $\chi(g)=1$ if ord $\chi(g)<\infty$. By the previous argument, the group $H:=\langle g\rangle$ is finite. Note that $H^{2}(M, \mathbf{Q})$ is dense in $H^{2}(M, \mathbf{R})$ and the positive cone $\mathcal{C}$ is open and non-empty in $H^{1,1}(M, \mathbf{R})$. Since the natural projection $p$ : $H^{2}(M, \mathbf{R}) \longrightarrow H^{1,1}(M, \mathbf{R})$ is continuous, there is then $\eta \in H^{2}(M, \mathbf{Q})$ such that $\eta^{(1,1)}:=p(\eta) \in \mathcal{C}$. Since $\mathcal{C}$ is a convex cone and stable under $\operatorname{Bir}(M)$, it follows that the element

$$
\tilde{\eta}:=\sum_{h \in H} h^{*} \eta
$$

is $H$-invariant, rational and satisfies $\tilde{\eta}^{(1,1)} \in \mathcal{C}$. Assume that $\chi(g) \neq 1$. Then, $\tilde{\eta}$ would have no $(2,0)$ and $(0,2)$ component, because it is $H$ invariant. That is, $\tilde{\eta}$ would be of pure $(1,1)$ type. Since $\tilde{\eta}$ is rational, we would then have $\tilde{\eta} \in N S(M) \otimes \mathbf{Q}$ by the Lefschetz (1,1)-Theorem. But $\left(\tilde{\eta}^{2}\right)>0$ by the choice of $\tilde{\eta}$, a contradiction to the negative definiteness of $N S(M)$. Thus $\chi(g)=1$.

q.e.d.

This theorem (together with the following examples) shows that the value $\chi(g)$ is an effective invariant which can distinguish certain nonprojective hyperkähler manifolds, especially those with elliptic NéronSeveri lattices, from projective ones.

Example 2.5. Let $S$ be a K3 surface having an automorphism $f$ with a Siegel disk $D$, say, $(D, f \mid D)$ is isomorphic to $\left(\Delta^{2}, F\right)$ with $F^{*}\left(z_{1}, z_{2}\right)=$ $\left(\alpha_{1} z_{1}, \alpha_{2} z_{2}\right)$. Here $\alpha_{1}$ and $\alpha_{2}$ are multiplicatively independent. Then, $\chi(f)=\alpha_{1} \alpha_{2}$ and $\chi(f)$ is not a root of unity. So, $N S(S)$ is elliptic by Theorem (2.4), and therefore the algebraic dimension $a(S)=0$ by the classification theory of surfaces (cf. [BHPV]).

We shall give examples of hyperkähler manifolds with similar automorphisms:

Example 2.6. Let $m \geq 2$ be an integer and $(S, f)$ be a McMullen's pair. Let $\left(M_{m}, f_{m}\right)$ be the pair of the Douady space $M_{m}:=S^{[m]}$ (cf. Introduction) and its automorphism $f_{m}$ naturally induced by $f$. Then, 
$M_{m}$ is a $2 m$-dimensional hyperkähler manifold such that $N S\left(M_{m}\right) \simeq$ $\langle-2(m-1)\rangle$. In particular, $N S\left(M_{m}\right)$ is elliptic and $\rho\left(M_{m}\right)=1$. Moreover, $\chi\left(f_{m}\right)$ is not a root of unity.

Proof. The first part is shown by [Be1]. The fact that $N S\left(M_{m}\right) \simeq$ $\langle-2(m-1)\rangle$ follows from $N S(S)=\{0\}$ and [ibid]. By [ibid], a symplectic from $\sigma_{M_{m}}$ of $M_{m}$ is given as follows: the 2 -from $\sum_{i=1}^{m} \operatorname{pr}_{i}^{*} \sigma_{S}$ on $\Pi_{1}^{m} S$ descends to the 2-form $\sigma_{S^{(m)}}$ on the Chow variety $S^{(m)}:=\left(\Pi_{i=1}^{m} S\right) / S_{m}$. The pullback of $\sigma_{S^{(m)}}$ under the Hilbert-Chow morphism $M_{m} \longrightarrow S^{(m)}$ gives $\sigma_{M_{m}}$.

By definition of $M_{m}$, the automorphism $f$ of $S$ naturally induces an automorphism $f_{m}$ of $M_{m}$. By the description of $\sigma_{M_{m}}$, one has $f_{m}^{*} \sigma_{M_{m}}=$ $\chi(f) \sigma_{M_{m}}$. Thus $\chi\left(f_{m}\right)=\chi(f)$ and is not a root of unity by Example $(2.5)$.

q.e.d.

The following corollary says that geometric action of $G(<\operatorname{Bir}(M))$ on $M$ can be approximated by its representation on $N S(M)$ when $N S(M)$ is hyperbolic or parabolic.

Corollary 2.7. Let $M$ be a hyperkähler manifold. Let $G<\operatorname{Bir}(M)$. Let $K:=\operatorname{Ker}\left(r_{N S}: G \longrightarrow \mathrm{O}(N S(M))\right)$ and $H:=r_{N S}(G)$. Assume that $N S(M)$ is hyperbolic or parabolic. Then $K$ is finite. In particular, $G$ is almost abelian iff so is $H$, and they have the same rank.

Proof. Let $G^{*}:=r(G)=\operatorname{Im}\left(r: G \longrightarrow \mathrm{O}\left(H^{2}(M, \mathbf{Z})\right)\right)$. By Theorem (2.3) and Proposition (8.3) in the appendix, it suffices to show that the group $K^{*}:=\left\{g \in G^{*}|g| N S(M)=i d\right\}$ is finite.

Consider the natural map $\tau: K^{*} \longrightarrow r_{T}(G) ; g \mapsto g \mid T(M)$.

By Theorem (2.4)(4)(5), $\left|r_{T}(G)\right|<\infty$. So, it suffices to show that $\tau$ is injective.

First consider the case where $N S(M)$ is hyperbolic. Then, $N S(M) \oplus$ $T(M)$ is of finite index in $H^{2}(M, \mathbf{Z})$. Thus, $\tau$ is injective.

Next consider the case where $N S(M)$ is parabolic. The remaing argument is quite similar to that of [Og1, Appendix].

By the assumption, we have $N S(M) \cap T(M)=\mathbf{Z} v$. This $v$ is primitive and satisfies $\left(v^{2}\right)=0$ and $v_{N S(M)}^{\perp}=N S(M)$ and $v_{T(M)}^{\perp}=$ $T(M)$. Then, the BF-form on $N S(M)$ descends to the bilinear form on $\overline{N S}(M):=N S(M) / \mathbf{Z} v$ and makes $\overline{N S}(M)$ a negative definite lattice of rank $\rho(M)-1$. Similarly, the BF-form on $T(M)$ descends to the bilinear form on $\bar{T}(M):=T(M) / \mathbf{Z} v$ and makes $\bar{T}(M)$ a non-degenerate lattice. The isometry $G^{*}$ also descends to the isometry of both $\overline{N S}(M)$ and $\bar{T}(M)$.

Let $\left\langle v, u_{i}(1 \leq i \leq \rho(M)-1)\right\rangle$ be an integral basis of $N S(M)$ and $\left\langle v, t_{j}(1 \leq j \leq k)\right\rangle$ be an integral basis of $T(M)$. Let $w \in H^{2}(M, \mathbf{Z})$ be an element such that

$$
\left\langle v, t_{j}(1 \leq j \leq k), u_{i}(1 \leq i \leq \rho(M)-1), w\right\rangle
$$


forms a basis of $H^{2}(M, \mathbf{Q})$. Let $g \in \operatorname{Ker} \iota$. Then we have

$$
\begin{gathered}
g(v)=v, g\left(u_{i}\right)=u_{i}, g\left(t_{j}\right)=t_{j} \\
g(w)=a(g) w+b(g) v+\sum_{i=1}^{\rho(M)-1} c_{i}(g) u_{i}+\sum_{j=1}^{k} d_{j}(g) t_{j} .
\end{gathered}
$$

Here $a(g), b(g), c_{i}(g)$ and $d_{j}(g)$ are rational numbers.

Note that $\left(v^{2}\right)=\left(v, u_{i}\right)=\left(v, t_{j}\right)=0$. Hence $(v, w) \neq 0$, because $H^{2}(X, \mathbf{Q})$ is non-degenerate. Thus $a(g)=1$ from $(w, v)=(g(w), g(v))$. From $\left(g(w), g\left(u_{l}\right)\right)=\left(w, u_{l}\right)$, we obtain

$$
\sum_{i=1}^{\rho(M)-1} c_{i}(g)\left(u_{i}, u_{l}\right)=0
$$

for all $l$ with $1 \leq l \leq \rho(M)-1$. Note that $\left\langle u_{i} \bmod \mathbf{Z} v\right\rangle_{i=1}^{\rho(M)-1}$ forms integral basis of $\overline{N S}(M)$ and recall that $\overline{N S}(M)$ is of negative definite. Thus, we have $c_{i}(g)=0$ for all $i$ from the equality above. Using the fact that $\left\langle t_{j} \bmod \mathbf{Z} v\right\rangle_{j=1}^{k}$ forms integral basis of $\bar{T}(M)$ and $\bar{T}(M)$ is nondegenerate, we also obtain that $d_{j}(g)=0$ for all $j$ in a similar manner. Thus $g(w)=w+b(g) v$. Since $\left(g(w)^{2}\right)=\left(w^{2}\right)$ and $(w, v) \neq 0$, we finally have $b(g)=0$. Hence, $g(w)=w$. Thus $g=i d_{H^{2}(M, \mathbf{Q})}$. Therefore $\tau$ is injective.

q.e.d.

\section{Salem polynomials and transcendental representations}

Throughout this section, $M$ is a hyperkähler manifold with elliptic $N S(M)$. We will study the transcendental representation of $\operatorname{Bir}(M)$ more closely by means of the notion of Salem polynomials. Our main result of this section is Theorem (3.4).

By Kronecker's theorem (see e.g., [Ta, Section 9.2]), a monic irreducible polynomial in $\mathbf{Z}[t]$ is cyclotomic iff all the roots are on the unit circle $S^{1}$. Salem polynomials form the second simplest class of monic irreducible polynomials in $\mathbf{Z}[t]$ in the view: "How many roots are off the unit circle?". The precise definition is:

Definition 3.1 (cf. [Mc], [GM]). An irreducible monic polynomial $\Phi(t) \in \mathbf{Z}[t]$ of degree $n$ is a Salem polynomial if the roots of $\Phi(t)$ consist of two real roots $\alpha$ and $1 / \alpha$ such that $\alpha>1$ and $n-2$ roots on the unit circle. The unique root $\alpha>1$ is the Salem number associated with $\Phi(t)$.

Remark 3.2. $\operatorname{deg} \Phi(t) \equiv 0(\bmod 2)$ and $\Phi(0)=1$ if $\Phi(t)$ is a Salem polynomial.

Recall that cyclotomic polynomials with fixed degree are at most finitely many (possibly empty). On the other hand, as it is shown by Gross and McMullen $[\mathbf{G M}]$, there are infinitely many Salem polynomials 
in each even degree $\geq 6$. The following finiteness criterion about Salem polynomials is in [Mc, Section 10]:

Proposition 3.3. There are at most finitely many Salem polynomials with fixed degree and bounded trace. That is, given an integer $n>$ 0 and real numbers $B_{1}<B_{2}$, there are at most finitely many Salem polynomials $\varphi(t)$ such that

$$
\operatorname{deg} \varphi(t)=n \text { and } B_{1}<\operatorname{tr} \varphi(t)<B_{2} .
$$

Here the trace of $\varphi(t)$ is the sum of roots counted with multiplicities, and is the same as the minus of the coefficient of the second leading term $t^{n-1}$.

Proof. The argument here is taken from [Mc, Section 10]. Write

$$
\varphi(t)=t^{n}+\sum_{k=1}^{n-1} a_{k} t^{k}+1 .
$$

Let $\alpha>1,1 / \alpha, \beta_{i}, \overline{\beta_{i}}(1 \leq i \leq(n-1) / 2)$ be the roots of $\varphi(t)$. Since $\beta_{i}$ are on the unit circle, one has

$$
-(n-1) \leq \sum_{i=1}^{(n-1) / 2} \beta_{i}+\overline{\beta_{i}} \leq n-1 .
$$

On the other hand, by the assumption, one has

$$
B_{1}<\operatorname{tr} \varphi(t)=\alpha+1 / \alpha+\sum_{i=1}^{(n-1) / 2} \beta_{i}+\overline{\beta_{i}}<B_{2} .
$$

Thus, the Salem number $\alpha>1$ is also bounded from above. Hence the roots of $\varphi(t)$ are in some compact set of $\mathbf{C}$ (independent of $\varphi(t)$ ). Up to sign, $a_{k}$ is the elementary symmetric function of degree $k$ of the roots. Thus $a_{k}$ are also in some compact set of $\mathbf{C}$ (independent of $\varphi(t)$ and $k$ ). Since $a_{k}$ are all integers, this implies the result. $\quad$ q.e.d.

As it is explained in the introduction, McMullen's pairs are closely related to Salem polynomials. Salem polynomials also play an important role in our study:

Theorem 3.4. Let $M$ be a hyperkähler manifold with elliptic $N S(M)$. Let $f \in \operatorname{Bir}(M)$. Set $F:=f^{*} \mid T(M)$. If ord $F=\infty$ (or equivalently ord $f=\infty$ by Theorem (2.4)(6)), then the characteristic polynomial

$$
\Phi(t):=\operatorname{det}(t I-F)
$$

of $F$ is a Salem polynomial. In particular, $\operatorname{rank} T(M) \equiv 0 \bmod 2$ and $f^{*} \mid T(M) \in \mathrm{SO}(T(M))$, if there is $f \in \operatorname{Bir}(M)$ with ord $f=\infty$. 
Proof. Recall that a complex number $\lambda$ is an eigenvalue of $F$ iff $\Phi(\lambda)=0$. We denote by $E(\lambda)$ the generalized $\lambda$-eigenspace of $F$, that is, $\Phi(\lambda)=0$ and

$$
E(\lambda):=\left\{v \in T(M) \otimes \mathbf{C} \mid(\lambda I-F)^{n} v=0 \exists n \in \mathbf{Z}_{>0}\right\} .
$$

We proceed with our proof by dividing it into several steps.

Claim 3.5. $\chi(f)$ is not a root of unity.

Proof. Otherwise, $\chi(f)=1$ by Theorem $(2.4)(6)$. Then $F=i d$ by Theorem $(2.4)(2)$, a contradiction. q.e.d.

Claim 3.6. $\Phi(t)$ has at least one root $\alpha_{0}$ outside the unit circle $S^{1}$, that is, there is a complex number $\alpha_{0}$ such that $\Phi\left(\alpha_{0}\right)=0$ and $\left|\alpha_{0}\right|>1$.

Proof. Note that $\operatorname{det} F= \pm 1$ by $F \in \mathrm{O}(T(M))$ and by the fact that $T(M)$ is non-degenerate if $N S(M)$ is elliptic. So, otherwise, the roots of $\Phi(t)$ would be all on $S^{1}$. On the other hand, they are algebraic integers. Hence all the roots of $\Phi(t)$ would the be roots of unity by Koronecker's theorem. In particular, $\chi(f)$ would be a root of unity, a contradiction to Claim (3.5).

q.e.d.

The next lemma, which follows from the Schwartz inequality, is also a special case of a well-known fact about the real orthogonal group $\mathrm{O}(p, q)$ (cf. [Mc, Lemma 3.1]):

Lemma 3.7. Let $g \in \mathrm{O}(1, m)$. Then $g$ has at most one eigenvalue (counted with multiplicity) outside $S^{1}$.

Claim 3.8. $\Phi(t)$ has exactly one real root $\alpha$ such that $\alpha>1$, and exactly two real roots, which are $\alpha$ and $1 / \alpha$, counted with multiplicities. The other roots are on the unit circle.

Proof. Set $S:=\oplus_{|\lambda|>1} E(\lambda)$. There is an $F$-stable $\mathbf{R}$-linear subspace $R \subset T(M) \otimes \mathbf{R}$ such that $S=R \otimes \mathbf{C}$; indeed, if $\lambda$ is an eigenvalue of $F$ with $|\lambda|>1$ and $v \in E(\lambda)$, then so is $\bar{\lambda}$ and $\bar{v} \in E(\bar{\lambda})$.

Choose $\alpha_{0}$ as in Claim (3.6). One has $0 \neq E\left(\alpha_{0}\right) \subset S$. Thus $R \neq\{0\}$. We show $\operatorname{dim}_{\mathbf{R}} R \leq 1$. Let $P$ be the positive 2-plane in $T(M) \otimes \mathbf{R}$ and $N$ the orthogonal complement of $P$ in $T(M) \otimes \mathbf{R}$. Then $R \subset N$ by $F(P)=P, F(N)=N$ and by the fact that $\chi(f)$ and $\overline{\chi(f)}$, the eigenvalues of $F \mid P$, are on $S^{1}$. On the other hand, since the signature of $N$ is $(1,0, t)$, one has $\operatorname{dim}_{\mathbf{R}} R \leq 1$ by Lemma $(3.7)$. So, $\operatorname{dim}_{\mathbf{R}} R=1$ by $R \neq\{0\}$. Thus $\Phi(t)$ has exactly one root $\alpha$ such that $|\alpha|>1$ and that this $\alpha$ is necessarily real and of multiplicity one.

Next we show that $\alpha>0$. Let $\mathcal{C}$ be the positive cone of $M$. Since $\alpha \in$ $\mathbf{R}$, there is $0 \neq v \in N$ such that $F(v)=\alpha v$. Note that $v \in H^{1,1}(M, \mathbf{R})$ by $N \subset H^{1,1}(M, \mathbf{R})$. One calculates $(v, v)=(F(v), F(v))=\alpha^{2}(v, v)$. Then $(v, v)=0$ by $|\alpha|>1$. So, replacing $v$ by $-v$ if necessary, one has 
$v \in \partial \mathcal{C}$. Here $\partial \mathcal{C}$ is the boundary of the positive cone. Since $f^{*}(\mathcal{C})=\mathcal{C}$, we have that $\alpha>0$. Thus $\alpha>1$ by $|\alpha|>1$.

Since the same holds for $f^{-1}$, it follows that $\Phi(t)$ has exactly one root $\beta$ inside $S^{1}$, and this $\beta$ is real, of multiplicity one, and satisfies $0<\beta<1$. In particular, the remaining roots $(\neq \alpha, \beta)$ of $\Phi(t)$ are on $S^{1}$. Now $\beta=1 / \alpha$ by $\operatorname{det} F= \pm 1$, and we are done. $\quad$ q.e.d.

Claim 3.9. $\Phi(t)$ is irreducible in $\mathbf{Z}[t]$.

Proof. Since $\Phi(t)$ is monic, it suffices to show that $\Phi(t)$ is irreducible in $\mathbf{Q}[t]$. By Claim (3.8), there is no $\varphi(t) \in \mathbf{Q}[t]$ such that $\Phi(t)=(\varphi(t))^{m}$ for $\exists m \geq 2$. So, the result follows from Theorem (2.4)(1). q.e.d.

The next claim completes the proof of Theorem (3.4).

Claim 3.10. $F \in \mathrm{SO}(T(M))$.

Proof. It is clear that $F \in \mathrm{O}(T(M))$. It suffices to show $\operatorname{det} F=$ 1. We already know that $\Phi(t) \in \mathbf{Z}[t]$ is an irreducible polynomial of $\operatorname{deg} \Phi(t)=\operatorname{rank} T(M) \geq 2$. Thus $\Phi( \pm 1) \neq 0$. So $\Phi(t)$ has no real root on the unit circle. Thus, the roots other than $\alpha, 1 / \alpha$ are all imaginary, and appear in pairs like $\lambda, \bar{\lambda}$. Hence the product of all the roots of $\Phi(t)$ is 1 and $\operatorname{det} F=1$.

q.e.d.

Now we are done. Q.E.D. of Theorem (3.4). $\quad$ q.e.d.

Corollary 3.11. Under the same assumption as in Theorem (3.4), each eigenvalue of $F$ is of multiplicity one, and $F$ is diagonalizable on $T(M) \otimes \mathbf{C}$.

Proof. This is because $\Phi(t)$ is irreducible.

q.e.d.

Remark 3.12. By Theorem (2.4)(6), the main part of Theorem (1.5)(1) follows once we will know $\operatorname{Im} \chi \simeq \mathbf{Z}$ when it is infinite. However, at the moment, even the finite generation of $\operatorname{Im} \chi$ is not so clear. For instance, $S^{1}\left(<\mathbf{C}^{\times}\right)$has a subgroup isomorphic to the additive group Q, namely

$$
\left\{w \in \mathbf{C}^{\times} \mid w=\exp (2 \pi \sqrt{-1} \sqrt{2} q), q \in \mathbf{Q}\right\} .
$$

The additive group $\mathbf{Q}$ is not finitely generated and any two elements of $\mathbf{Q}$ are not linearly independent over $\mathbf{Z}$. Also, $S^{1}$ has a subgroup isomorphic to the additive group $\mathbf{Z}^{r}$ for each $r>0$. For instance, one can check that the following subgroup is isomorphic to $\mathbf{Z}^{r}$ :

$$
\left\{w \in \mathbf{C}^{\times} \mid w=\exp \left(2 \pi \sqrt{-1}\left(\sum_{k=1}^{r} a_{k} n_{k}\right)\right),\left(n_{k}\right)_{k=1}^{r} \in \mathbf{Z}^{r}\right\},
$$

where $a_{k}:=\log _{2} p_{k}$ and $p_{k}$ are mutually different odd prime numbers. We will study finite generation of $\operatorname{Im} \chi$ and its rank in the next section. 


\section{Automorphism group of a hyperkähler manifold with elliptic Néron-Severi lattice}

In this section, we shall prove the main part of Theorem $(1.5)(1)$ dividing into several steps. Existence part in dimension 2 will be shown in Section 6. Cruicial steps are Propositions (4.3) and (4.4).

Let $M$ be a hyperkähler manifold with elliptic $N S(M)$. Put:

$$
A^{\prime}:=\operatorname{Im}\left(\chi: \operatorname{Bir}(M) \longrightarrow \mathbf{C}^{\times}\right) \text {and } A:=\operatorname{Im}(\operatorname{Bir}(M) \longrightarrow S O(T(M))) \text {. }
$$

By Theorem (2.4)(2), there is a natural isomorphism:

$$
\psi: A \simeq A^{\prime} ; g^{*} \mid T(M) \mapsto \chi(g) .
$$

As we remarked in (3.12), we may show that $A^{\prime} \simeq \mathbf{Z}$, assuming that there is $f \in \operatorname{Bir}(M)$ such that

$$
\text { ord } F=\infty \text {, where } F:=f^{*} \mid T(M) .
$$

Let $\Phi(t)$ be the characteristic polynomial of $F$. We already know that $\Phi(t)$ is a Salem polynomial (Theorem (3.4)). Thus $\operatorname{deg} \Phi(t)=$ $\operatorname{rank} T(M)$ must be even. This already proves the second statement of $(1.5)(1)$.

Let us compare $F$ with another element $G:=g^{*} \mid T(M) \in A$ given by $g \in \operatorname{Bir}(M)$.

Claim 4.1. There is a polynomial $\varphi(t) \in \mathbf{Q}[t]$ such that $G=\varphi(F)$.

Proof. Choose an integral basis of $T(M)$. Then, we may regard $F, G \in M(n, \mathbf{Q})$, where $n:=\operatorname{rank} T(M)$. Since $A$ is an abelian group, one has $F G=G F$. Thus $G$ is an element of the $\mathbf{Q}$-linear subspace $V$ of $M(n, \mathbf{Q})$. Here

$$
V:=\{X \in M(n, \mathbf{Q}) \mid F X=X F\} .
$$

The equation $F X=X F$ is a system of linear homogeneous equations with rational coefficients. One can solve this by Gauss' elimination method, which obviously commutes with field extensions. Thus

$$
V \otimes \mathbf{C}=\{X \in M(n, \mathbf{C}) \mid F X=X F\} .
$$

By Corollary (3.11), there is a basis $\left\langle v_{k}\right\rangle_{k=1}^{n}$ of $T(M) \otimes \mathbf{C}$ under which $F$ is represented by a diagonal matrix $F=\operatorname{diag}\left(a_{1}, \cdots, a_{n}\right)$ with $a_{i} \neq a_{j}$ for $i \neq j$. Identify $X$ with its matrix representation with respect to $\left\langle v_{k}\right\rangle_{k=1}^{n}$. Now by an explicit matrix calculation, one sees $V \otimes \mathbf{C}=D(n)$. Here $D(n)$ is the set of diagonal matrices of size $n$. Thus $\operatorname{dim}_{\mathbf{C}} V \otimes \mathbf{C}=n$, and hence $\operatorname{dim}_{\mathbf{Q}} V=n$. Since $\Phi(t)$ is irreducible in $\mathbf{Q}[t]$, the $n$-elements $A^{k}(0 \leq k \leq n-1)$ of $V$ are linearly independent over $\mathbf{Q}$ and then form a Q-basis of $V$. Since $G \in V$, one has then $G=\varphi(F)$ for $\exists \varphi(t) \in \mathbf{Q}[t]$ (with $\operatorname{deg} \varphi(t) \leq n-1$ ).

q.e.d. 
Claim 4.2. Let $K(\subset \mathbf{C})$ be the minimal splitting field of $\Phi(t)$ and $O_{K}$ be the ring of integers of $K$, i.e., the normalization of $\mathbf{Z}$ in $K$, and $U_{K} \subset O_{K}$ be the group of units of $O_{K}$. Then, $\chi(g) \in U_{K}$ for $\forall g \in \operatorname{Aut}(M)$.

Proof. $\chi(g)$ and $\overline{\chi(g)}$ are eigenvalues of $G$. Thus, by Claim (4.1), $\chi(g), \overline{\chi(g)} \in K . \chi(g)$ and $\overline{\chi(g)}$ are also roots of the monic polynomial $\operatorname{det}(t I-G) \in \mathbf{Z}[t]$. Thus $\chi(g), \overline{\chi(g)} \in O_{K}$. By Theorem (2.4)(3), we have also $1=|\chi(g)|^{2}=\chi(g) \overline{\chi(g)}$. Hence $\chi(g), \overline{\chi(g)} \in U_{K}$. q.e.d.

Proposition 4.3. A is a finitely generated free abelian group.

Proof. By Claim (4.2), one has $A^{\prime}<\mathbf{C}^{\times} \cap U_{K}<U_{K}$. By Dirichlet's unit theorem (see e.g., [Ta, Section 9.3]), $U_{K}$ is a finitely generated abelian group. Hence, so is its subgroup $A^{\prime}$ by the fundamental theorem of finitely generated abelian groups. Moreover, $A^{\prime}$ is torsion free by Theorem $(2.4)(6)$. Now the result follows from $A \simeq A^{\prime}$ (Theorem $(2.4)(2))$.

q.e.d.

Set $r:=\operatorname{rank} A$. The next proposition completes the proof of the main part of Theorem (1.5)(1):

Proposition 4.4. $r=1$ (if $r>0$ ).

Proof. Assuming that $r \geq 2$, we shall derive a contradiction. Take a free basis $\left\langle F_{i}:=f_{i} \mid T(M)\right\rangle_{i=1}^{r}$ of $A$. We may now re-choose $f$ so that $f=f_{1}$. Since $r \geq 2$, there is $f_{2}$. Set $g:=f_{2}$. As before, we write $F=f^{*} \mid T(M)$ and $G=g^{*} \mid T(M)$. One has ord $F=$ ord $G=\infty$, because $F$ and $G$ form a part of the free basis of $A$. Thus, the characteristic polynomials $\Phi_{F}(t)$ and $\Phi_{G}(t)$ of $F$ and $G$ are both Salem polynomials (Theorem (3.4)). Let $\alpha>1$ and $\beta>1$ be the Salem numbers of $\Phi_{F}(t)$ and $\Phi_{G}(t)$.

Let $(n, m) \in \mathbf{Z}^{2}-\{(0,0)\}$. Then ord $F^{n} G^{m}=\infty$, because $F$ and $G$ form a part of free basis of $A$. Thus, the characteristic polynomial $\varphi^{(n, m)}(t)$ of $F^{n} G^{m}$ is also a Salem polynomial (Theorem (3.4)). In particular, $\varphi^{(n, m)}(t)$ has exactly two roots off the unit circle and they are both real. On the other hand, $F G=G F$ and $F$ and $G$ are both diagonalizable by Corollary (3.11). Thus $F$ and $G$ are simultaneously diagonalizable. Hence the pair of the two (necessarily real) roots of $\varphi^{(n, m)}(t)$ off the unit circle must be either

$$
\left\{\alpha^{n} \beta^{m}, \alpha^{-n} \beta^{-m}\right\} \text { or }\left\{\alpha^{n} \beta^{-m}, \alpha^{-n} \beta^{m}\right\} \text {. }
$$

By replacing $g=f_{2}$ by $g=f_{2}^{-1}$ if necessary, one can arrange so that the pairs are the first ones $\left\{\alpha^{n} \beta^{m}, \alpha^{-n} \beta^{-m}\right\}$ for $\forall(n, m) \in \mathbf{Z}^{2}-\{(0,0)\}$.

Claim 4.5. There are real numbers $1<C_{1}<C_{2}$ such that $\left|\mathcal{T}_{0}\right|=\infty$. Here

$$
\mathcal{T}_{0}:=\left\{(n, m) \in \mathbf{Z}^{2}-\{(0,0)\} \mid C_{1}<\alpha^{n} \beta^{m}<C_{2}\right\} .
$$


Proof. Since $\alpha, \beta, C_{1}, C_{2}>1$, the inequalty in $\mathcal{T}_{0}$ is equivalent to

$$
\text { (*) } \log C_{1}-n \log \alpha<m \log \beta<\log C_{2}-n \log \alpha .
$$

Choose $1<C_{1}<C_{2}$ such that $\beta<C_{2} / C_{1}$, i.e., $0<\log \beta<\log C_{2}-$ $\log C_{1}$. For each $n \in \mathbf{Z}_{<0}$, both sides of $(*)$ are then positive, of distance $>\log \beta$. Thus, for each such $n$, there is at least one $m \in \mathbf{Z}_{>0}$ satisfying $(*)$. This implies the result.

q.e.d.

We can now complete the proof of Proposition (4.4). Let us choose $C_{1}$ and $C_{2}$ as in Claim (4.5). Let $(n, m) \in \mathcal{T}_{0}$. Then the roots of $\varphi^{(n, m)}(t)$ are:

$$
\alpha^{n} \beta^{m} \alpha^{-n} \beta^{-m} \gamma_{i} \overline{\gamma_{i}} .
$$

Here $1 \leq i \leq(\operatorname{rank} T(M)-2) / 2$ and $\gamma_{i}$ are on the unit circle. One has

$$
C_{1}<\alpha^{n} \beta^{m}<C_{2} \text { and } 1 / C_{2}<\alpha^{-n} \beta^{-m}<1 / C_{1} .
$$

Thus $\operatorname{tr} \varphi^{(n, m)}(t)$ are bounded by the constants (independent on $(n, m) \in$ $\left.\mathcal{T}_{0}\right)$. Hence, by Proposition (3.3), there is a set $\mathcal{S}$ of polynomials such that $|\mathcal{S}|<\infty$ and

$$
\varphi^{(n, m)}(t) \in \mathcal{S} \text { for } \forall(n, m) \in \mathcal{T}_{0} .
$$

By $\left|\mathcal{T}_{0}\right|=\infty$ and $|\mathcal{S}|<\infty$, there is $\Psi(t) \in \mathcal{S}$ such that $\left|\mathcal{T}_{1}\right|=\infty$. Here

$$
\mathcal{T}_{1}:=\left\{(n, m) \in \mathcal{T}_{0} \mid \varphi^{(n, m)}(t)=\Psi(t)\right\} .
$$

The polynomial $\Psi(t)$ has at most finitely many roots. On the other hand, $\chi\left(f^{n} g^{m}\right)$ is a root of $\varphi^{(n, m)}(t)$. Thus there is a root $\delta$ of $\Psi(t)$ such that $\left|\mathcal{T}_{2}\right|=\infty$. Here

$$
\mathcal{T}_{2}:=\left\{(n, m) \in \mathcal{T}_{1} \mid \chi\left(f^{n} g^{m}\right)=\delta\right\} .
$$

Choose one $\left(n_{0}, m_{0}\right) \in \mathcal{T}_{2}$. Then for each $(n, m) \in \mathcal{T}_{2}$, one has

$$
\chi\left(f^{n} g^{m}\right)=\chi\left(f^{n_{0}} g^{m_{0}}\right) \text {, i.e., } \chi\left(f^{n-n_{0}} g^{m-m_{0}}\right)=1 .
$$

Then $F^{n-n_{0}} G^{m-m_{0}}=i d$ by $A \simeq A^{\prime}$. Since $F$ and $G$ form a part of free basis of $A$, it follows that $n=n_{0}$ and $m=m_{0}$, i.e., $\mathcal{T}_{2}=\left\{\left(n_{0}, m_{0}\right)\right\}$, a contradiction to $\left|\mathcal{T}_{2}\right|=\infty$.

Hence, $r=1$ if $r>0$. This completes the proof of Proposition (4.4) and therefore the proof of the main part of Theorem (1.5)(1).

It remains to show the existence of $M$ with $r=1$ and 0 . By the main part of Theorem $(1.5)(1)$, the pair $\left(M_{m}, f_{m}\right)(m \geq 2)$ in Example (2.6) satisfies $r=1$ as well as other necessary requirements. In addition, the hyperkähler manifold $D_{m}$ in Corollary (1.7) satisfies $r=0$ as well as other necessary requirements. A 2-dimensional example with $r=0$ will be given in Section 6 . 
Remark 4.6. Here the Dirichlet unit theorem, one of two most fundamental theorems in algebraic number theory, played a crucial role in the proof. It may be interesting to seek a relation hidden between hyperkähler manifolds and the other fundamental theorem, the finiteness of class numbers (if any). See $[\mathbf{H L O Y}]$ for one of such relations in dimension 2 .

\section{Automorphism group of a hyperkähler manifold with parabolic Néron-Severi lattice}

In this section we shall prove the main part of Theorem (1.5)(2). We freely use some basic properties of almost abelian groups. They are in Section 8. Optimality of the estimate in dimension 2 will be shown in Section 7. Throughout this section $M$ is a hyperkähler manifold with parabolic $N S(M)$.

Set $H:=\operatorname{Im}\left(r_{N S}: \operatorname{Bir}(M) \longrightarrow \mathrm{O}(N S(M))\right)$. By Corollary $(2.7)$ and Proposition (8.3), we may show that $H$ is almost abelian of rank at most $\rho(X)-1$. However, this follows from the next purely lattice theoretical:

Proposition 5.1. Let $L$ be a parabolic lattice of rank $r$. Let $N<$ $\mathrm{O}(L)$. Then $N$ is an almost abelian group of rank at most $r-1$.

Proof. Let $v$ be the unique (up to sign) primitive totally isotoropic element of $L$. Then $g(v)= \pm v$ for each $g \in N$, and those $g$ with $g(v)=v$ form a subgroup of $N$ of index at most 2. So, by Proposition (8.3), we may assume that $g(v)=v$ for all $g \in N$.

The bilinear form of $L$ descends to the bilinear form on $\bar{L}:=L / \mathbf{Z} v$ and makes $\bar{L}$ a negative definite lattice of rank $r-1$. Our isometry $N$ also descends to the isometry of $\bar{L}$, say $g \mapsto \bar{g}$.

Set

$$
N^{(0)}:=\operatorname{Ker}(N \longrightarrow \mathrm{O}(\bar{L}) ; g \mapsto \bar{g}) .
$$

By the negative definteness of $\bar{L}$, we have $\left[N: N^{(0)}\right]<\infty$. So, again by Proposition (8.3), it now suffices to show that $N^{(0)}$ is almost abelian of rank at most $r-1$.

Let $\left\langle v, u_{i}(1 \leq i \leq r-1)\right\rangle$ be an integral basis of $L$. Let $g \in N^{(0)}$. Then we have

$$
g(v)=v, g\left(u_{i}\right)=u_{i}+\alpha_{i}(g) v .
$$

Here $\alpha_{i}(g)(1 \leq i \leq r-1)$ are integers uniquely determined by $g$. It is easy to see that the following map $\varphi$ is a group homomorphism:

$$
\varphi: N \longrightarrow \mathbf{Z}^{r-1} ; g \mapsto\left(\alpha_{i}(g)\right)_{i=1}^{r-1}
$$

This $\varphi$ is clearly injective by the form of $g$. Thus the result follows. q.e.d. 
Remark 5.2. It might be more desirable to give a more geometric argument. It is possible in dimension 2. Indeed, if $S$ is a K3 surface with parabolic $N S(S)$, then the algebraic dimension $a(S)=1$ and $S$ admits the unique elliptic fibration $a: S \longrightarrow \mathbf{P}^{1}$ (the algebraic reduction). This $f$ is $\operatorname{Aut}(S)$-stable. Moreover, as it is easily seen, $f$ admits at least three singular fibers (cf. [VZ]). Thus the group $K:=\operatorname{Ker}(\operatorname{Aut}(S) \longrightarrow$ Aut $\left.\left(\mathbf{P}^{1}\right)\right)$ is of finite index in Aut $(S)$. Since $f$ has no horizontal curve by $a(S)=1$, the group $K$ acts on each fiber as translations. Thus, $K$ can be embedded into the Mordell-Weil group of the Jacobian fibration $j: J \longrightarrow \mathbf{P}^{1}$ of $f$. The surface $J$ is an algebraic K3 surface. Therefore $K$ is an abelian group of rank at most 18 (cf. [Sh]). However, it seems difficult to generalize this argument in higher dimensional case, because almost nothing is known about the algebraic dimension and the structure of the algebraic reduction maps of $M$.

\section{Automorphism group of a McMullen's K3 surface}

In this section we shall prove Corollary (1.6) and the existence part in dimension 2 of Theorem (1.5)(1). Let $(S, f)$ be a McMullen's pair. Then $r=1$ by the main part of Theorem (1.5)(1). First, we shall show that Aut $(S) \simeq \mathbf{Z}$. Again by the main part of Theorem (1.5)(1), this follows from the next Proposition which we learned from Professor JongHae Keum:

Proposition 6.1. Let $S$ be a K3 surface with $\rho(S)=0$. Let $g \in$ Aut $(S)$. Then $g=$ id iff $\chi(g)$ is a root of unity.

Proof. Only if part is trivial. Assume that $\chi(g)$ is a root of unity. Then $\chi(g)=1$ by Theorem (2.4)(6) applied for $\langle g\rangle$. Thus $g^{*} \mid T(S)=i d$ by Theorem $(2.4)(2)$, and hence $g^{*}=i d$ on $H^{2}(S, \mathbf{Z})$ by $\rho(S)=0$. Then one has $g=i d$ by the global Torelli theorem for K3 surfaces (see for instance $[\mathbf{B H P V}])$.

q.e.d.

The following proposition and Proposition (6.1) complete the proof of the rest:

Proposition 6.2. There are at most countably many K3 surfaces with $\rho(S)=0$ and having an automorphism $f$ such that $\chi(f)$ is not a root of unity. In particular, in the (20-dimensional) period domain, K3 surfaces such that $\rho(S)=0$ and Aut $(S)=\{i d\}$ form the complement of the union of countably many rational hyperplanes and at most countably many points.

Proof. By the surjectivity of the period mapping and the Lefschetz $(1,1)$-Theorem, K3 surfaces with $\rho(S)=0$ form the complement of the union of the countably many rational hyperplanes in the period domain. Thus, the last statement follows from the first statement and Proposition (6.1). 
Let us show the first statement. The following argument is similar to $[\mathbf{M c}]$. Let $S$ be a K3 surface with $\rho(S)=0$ and with $f \in \operatorname{Aut}(S)$ such that $\chi(f)$ is not a root of unity. For each such $S$, let us choose a marking $\iota_{S}: T(S)=H^{2}(S, \mathbf{Z}) \simeq \Lambda$, where $\Lambda$ is the K3 lattice (cf. Section 7). The characteristic polynomial of $\iota_{S} \circ f^{*} \circ \iota_{S}^{-1}$ must be a Salem polynomial by Theorem (3.4). There are at most countably many Salem polynomials of degree 22 . So, there are at most countably many element $g \in \mathrm{O}(\Lambda)$ whose characteristic polynomials are Salem polynomials. By Corollary (3.11), each eigenvalue of such $g$ is of multiplicity one and the corresponding eigenspaces are one-dimensional. Thus, there are only countably many eigenspaces of all such $g$. On the other hand, the possible 1-dimensional subspaces $\iota_{S}\left(\mathbf{C} \sigma_{S}\right) \subset \Lambda \otimes \mathbf{C}$ must be eigenspaces of one of such $g$. Thus, the subspaces $\iota_{S}\left(\mathbf{C} \sigma_{S}\right)$ are at most countably many as well. Then, by the global Torelli Theorem, there are at most countably many such $S$.

q.e.d.

\section{Non-projective K3 surface of large automorphism group}

In this section, we shall complete the proof of Theorem $(1.5)(2)$ by showing the following:

Theorem 7.1. There is a K3 surface $S$ such that $a(S)=1, \rho(S)=$ 19 and such that Aut $(S)$ is an almost abelin group of rank $18(=19-1)$. In particular, the estimate in Theorem (1.5)(2) is optimal in dimension 2 .

It is well-known that $a(S)=1$ iff $N S(S)$ is parabolic, and $\rho(S) \leq 19$ if $a(S) \leq 1$. On the other hand, by the main part of Theorem (1.5), Aut $(S)$ is almost abelian of rank at most $\max (\rho(S)-1,1)$ if $a(S) \leq$ 1. So, the K3 surface $S$ in Theorem (7.1) is also a non-projective K3 surface having maximal possible automorphism group (up to finite group factors).

In what follows, we shall show Theorem (7.1), dividing the proof into several steps. Unfortunately, since our proof is based on the surjectivity of the period mapping and the global Torelli Theorem, our K3 surface and the group action are not so geometrically "visible".

Let

$$
\Lambda:=\Lambda_{\mathrm{K} 3}:=U^{\oplus 3} \oplus E_{8}(-1)^{\oplus 2}
$$

be the K3 lattice. Let $i \in\{0,1,2\}$ and $\left\langle e_{i}, f_{i}\right\rangle$ be the integral basis of the $(i+1)-$ th $U$ in $\Lambda$ s.t. $\left(e_{i}^{2}\right)=\left(f_{i}^{2}\right)=0,\left(e_{i}, f_{i}\right)=0$, and $\left\langle v_{i j}\right\rangle_{j=1}^{8}$ be an integral basis of the $i$-th $E_{8}(-1)(i=1,2)$ in $\Lambda$, which forms the Dynkin diagram of type $E_{8}$. (We need not specify the position of each $v_{i j}$ in the Dynkin diagram.)

Choose "sufficiently large" mutually different primes numbers $p, q$, $p_{i}, q_{i}(1 \leq i \leq 8)$. (The term "sufficiently large" will be clear in the 
proof of the next lemma.) We then define the sublattices $\bar{N}, N$ and $L$ of $\Lambda$ by:

$$
\begin{gathered}
\bar{N}:=\mathbf{Z}\left\langle e_{1}-p f_{1}\right\rangle \oplus \mathbf{Z}\left\langle e_{2}-q f_{2}\right\rangle \oplus \mathbf{Z}\left\langle e_{1}-p_{1 j} v_{1 j}\right\rangle_{j=1}^{8} \oplus \mathbf{Z}\left\langle e_{2}-q_{2 j} v_{2 j}\right\rangle_{j=1}^{8}, \\
N:=\mathbf{Z}\left\langle e_{0}\right\rangle \oplus \bar{N}, \\
L:=N+\mathbf{Z}\left\langle f_{0}\right\rangle=U \oplus \bar{N} .
\end{gathered}
$$

Lemma 7.2. $N$ and $L$ satisfy:

(1) $N$ is parabolic of rank 19, and $L$ is a hyperbolic lattice of rank 20.

(2) $N$ and $L$ are primitive in $\Lambda$.

(3) $N$ does not represent -2 , i.e., there is no $x \in N$ with $\left(x^{2}\right)=-2$.

Proof. Note that $\bar{N}$ is elliptic of rank 18 and that $\left(e_{0}^{2}\right)=\left(e_{0}, \bar{N}\right)=0$. Thus, $N$ is parabolic of rank 19. (This does not depend on the choice of $p, q, p_{i}, q_{i}$ whenever they are positive integers.) Proof for $L$ is almost the same and we omit it.

Next we shall show that $N$ is primitive in $\Lambda$. (Proof for $L$ is the same and we omit it.) Here, we use the fact that $p, q, p_{i}, q_{i}$ are mutually different prime numbers (but do not yet use the assumption they are "sufficiently large"). Suppose that there are rational numbers $x_{0}, x_{1}$, $x_{2}, x_{1 i}, x_{2 i}$ such that

$$
\begin{aligned}
x_{0} e_{0}+x_{1}\left(e_{1}-p f_{1}\right) & +x_{2}\left(e_{2}-q f_{2}\right) \\
& +\sum_{j=1}^{8} x_{1 j}\left(e_{1}-p_{1 j} v_{1 j}\right)+\sum_{j=1}^{8} x_{2 j}\left(e_{2}-p_{2 j} v_{2 j}\right) \in \Lambda .
\end{aligned}
$$

We need to show that $x_{0}, x_{1}, x_{2}, x_{1 j}, x_{2 j}$ are all integers.

Calculate that

$$
\begin{aligned}
& x_{0} e_{0}+x_{1}\left(e_{1}-p f_{1}\right)+x_{2}\left(e_{2}-q f_{2}\right) \\
& \quad+\sum_{j=1}^{8} x_{1 j}\left(e_{1}-p_{1 j} v_{1 j}\right)+\sum_{j=1}^{8} x_{2 j}\left(e_{2}-p_{2 j} v_{2 j}\right) \\
& =x_{0} e_{0}+\left(x_{1}+\sum_{j=1}^{8} x_{1 j}\right) e_{1}-x_{1} p f_{1}+\left(x_{2}+\sum_{j=1}^{8} x_{2 j}\right) e_{2} \\
& \quad-x_{2} q f_{2}-\sum_{j=1}^{8} x_{1 j} p_{j} v_{1 j}-\sum_{j=1}^{8} x_{2 j} q_{j} v_{2 j} .
\end{aligned}
$$

Since $e_{0}, e_{1}, f_{1}, e_{2}, f_{2}, v_{1 j}, v_{2 j}$ form a part of free basis of $\Lambda$, the coefficients of the second line are all integers:

$$
\begin{aligned}
& x_{0}, x_{1} p, x_{2} q, x_{1 j} p_{j}, x_{2 j} q_{j} \in \mathbf{Z} ; \\
& x_{1}+\sum_{j=1}^{8} x_{1 j}, x_{2}+\sum_{j=1}^{8} x_{2 j} \in \mathbf{Z} .
\end{aligned}
$$


Thus $x_{0} \in \mathbf{Z}$ and one can write $x_{1}=c_{1} / p, x_{2}=c_{2} / q, x_{1 j}=c_{1 j} / p_{j}$, $x_{2 j}=c_{2 j} / q_{j}$, where $c_{*}$ and $c_{* *}$ are integers. By substituting these into the second two quantities, one obtains

$$
\frac{c_{1}}{p}+\sum_{j=1}^{8} \frac{c_{1 j}}{p_{j}}, \frac{c_{2}}{q}+\sum_{j=1}^{8} \frac{c_{2 j}}{q_{j}} \in \mathbf{Z} .
$$

By clearing the denominator of the first quantity, one has

$$
p p_{1} \cdots p_{8} \mid\left(c_{1} p_{1} \cdots p_{8}+\sum_{j=1}^{8} c_{1 j} p p_{1} \cdots p_{j-1} p_{j+1} \cdots p_{8}\right) .
$$

Since $p$ divides the second sum, we have $p \mid c_{1} p_{1} \cdots p_{8}$, and therefore $p \mid c_{1}$. Similarly, $p_{j}$ must divide the term $c_{1 j} p p_{1} \cdots p_{j-1} p_{j+1} \cdots p_{8}$, whence $p_{j} \mid c_{1 j}$. For the same reason, one has also $q \mid c_{2}$ and $q_{j} \mid c_{2 j}$. Hence, the coefficients $x_{0}, x_{1}, x_{2}, x_{1 j}, x_{2 j}$ are all integers.

Let us show the assertion (3). Here we use the fact that $p, q, p_{i}, q_{i}$ are "sufficiently large". Since $E_{8}(-1)$ is negative definite, there are only finitely many elements $r$ with $\left(r^{2}\right)=-2$. (As is well known, there are exactly 240 such elements, but we do not need this precise number.) Then, there is no $(-2)$-element of the form $\sum_{j=1}^{8} x_{i j} n_{j} v_{i j}\left(x_{i j} \in \mathbf{Z}\right)$ if $n_{j}(1 \leq j \leq 8)$ are sufficiently large, say $n_{j}>C$ for $\forall j$. Choose 8 prime numbers $p_{j}, q_{j}$ so that they are larger than such $C$.

Let $v \in N$ and write $v$ under the integral basis as:

$$
\begin{aligned}
v= & x_{0} e_{0}+x_{1}\left(e_{1}-p f_{1}\right)+x_{2}\left(e_{2}-q f_{2}\right) \\
& +\sum_{j=1}^{8} x_{1 j}\left(e_{1}-p_{j} v_{1 j}\right)+\sum_{j=1}^{8} x_{2 j}\left(e_{2}-q_{j} v_{2 j}\right) .
\end{aligned}
$$

Then, by using $\left(e_{i}, v_{i j}\right)=\left(e_{i}^{2}\right)=0$, one calculates:

$$
\left(v^{2}\right)=-2 p x_{1}^{2}-2 q x_{2}^{2}+\left(\sum_{j=1}^{8} x_{1 j} p_{j} v_{1 j}\right)^{2}+\left(\sum_{j=1}^{8} x_{2 j} q_{j} v_{2 j}\right)^{2} .
$$

Here each of the four summands in $\left(v^{2}\right)$ is even and 0 or strictly less than -2 by our choice of $p, q, p_{j}, q_{j}$. Thus $\left(v^{2}\right) \neq-2$. This completes the proof.

q.e.d.

As usual, by a marked K3 surface, we mean a pair $(S, \iota)$ of a K3 surface $S$ and an isometry $\iota: H^{2}(S, \mathbf{Z}) \simeq \Lambda$.

Proposition 7.3. There is a marked $K 3$ surface $(S, \iota)$ such that $\iota(N S(S))=N$.

Proof. Put

$$
T:=N_{\Lambda}^{\perp}=\mathbf{Z} e_{0} \oplus \bar{N}_{U^{\oplus 2} \oplus E_{8}(-1)^{\oplus 2}}^{\perp}=\mathbf{Z} e_{0} \oplus \bar{T} .
$$


Then $\bar{T}$ is a positive definite lattice of rank 2. Choose an integral basis $\left\langle u_{1}, u_{2}\right\rangle$ of $\bar{T}$. Put $\left(u_{1}, u_{1}\right)=2 a,\left(u_{2}, u_{2}\right)=2 c,\left(u_{1}, u_{2}\right)=b$, and $A:=$ $4 a c-b^{2}$. Then $a>0$ and $A>0$. Consider the element $\sigma$ of $T_{\mathbf{C}}$ defined by

$$
\sigma=\sqrt{2} e_{0}+\frac{-b+\sqrt{A} i}{2 a} u_{1}+u_{2} i=\sqrt{-1} .
$$

It is easy to check that

$$
(\sigma, \sigma)=0,(\sigma, \bar{\sigma})=\frac{A}{a}>0 .
$$

Thus, by the sujectivity of the period mapping of K3 surfaces (see e.g., $[\mathbf{B H P V}])$, there is a marked K3 surface $(S, \iota)$ such that $\iota\left(\sigma_{S}\right)=\sigma$. Note that $N=T_{\Lambda}^{\perp}$. Then the equality $\iota(N S(S))=N$ follows from the next lemma.

q.e.d.

Lemma 7.4. Under the identification of $H^{2}(S, \mathbf{Z})$ with $\Lambda$ by $\iota$, the lattice $T$ is the transcendental lattice of $S$.

Proof. Let $M$ be the minimal primitive sublattice of $\Lambda$ such that $\sigma \in M_{\mathbf{C}}$. It is clear that $M \subset T$. We need to show that $T \subset M$. Since $\sigma-\bar{\sigma} \in M_{\mathbf{C}}$, we have $(\sqrt{A} i / a) u_{1} \in M_{\mathbf{C}}$. Thus $u_{1} \in M$ (by the primitivity of $M$ ), and therefore $\sqrt{2} e_{0}+u_{2} \in M_{\mathbf{C}}$ by $\sigma \in M_{\mathbf{C}}$. Since $e_{0}, u_{2} \in \Lambda$, this implies $\sqrt{2} e_{0}+u_{2} \in M_{\mathbf{Q}(\sqrt{2})}$, and therefore $-\sqrt{2} e_{0}+u_{2} \in$ $M_{\mathbf{Q}(\sqrt{2})}$ by the Galois theory. Thus $e_{0}, u_{2} \in M$ again by the primitivity of $M$. Hence $T \subset M$.

q.e.d.

The next theorem will complete the proof.

Theorem 7.5. Let $S$ be a K3 surface in Proposition (7.3). Then $a(S)=1, \rho(S)=19$ and Aut $(S)$ is an almost abelian group of rank 18 .

In what follows, we identify $H^{2}(S, \mathbf{Z})$ with $\Lambda$ by the marking $\iota$ and use the notation in Proposition (7.3) and its proof. Then $N S(S)=N$ and $T(S)=T$. Thus $a(S)=1$ and $\rho(S)=19$. Let us consider $\operatorname{Aut}(S)$. Let $L$ be the hyperbolic lattice defined just before Lemma (7.2). We have $L_{\Lambda}^{\perp}=\bar{T}$.

Lemma 7.6. For each $i(1 \leq i \leq 18)$, there is an isometry $\varphi_{i}$ of $L$ of the following form:

$$
\begin{gathered}
e_{0} \mapsto e_{0}, f_{0} \mapsto f_{0}+\gamma_{i} e_{0}+\sum_{k=1}^{18} c_{i k} w_{i k} ; \\
w_{i} \mapsto w_{i}+m w_{i} ; w_{j} \mapsto w_{j}(j \neq i) .
\end{gathered}
$$

Proof. Put $Q:=\left(\left(w_{k}, w_{l}\right)\right)_{1 \leq k, l \leq 18}$. This is a negative definite integral matrix. Put $m=\operatorname{det} Q$. Then $\varphi_{i}$ induces an isometry of $N$ and satisfies 
$\left(\varphi_{i}\left(f_{0}\right), \varphi_{i}\left(e_{0}\right)=\left(f_{0}, e_{0}\right)\right.$ (regardless of values $\left.\gamma_{i}, c_{i k}\right)$. Now it suffices to find $\gamma_{i}, c_{i k} \in \mathbf{Z}$ such that

$$
\left(\varphi_{i}\left(f_{0}\right), \varphi_{i}\left(w_{k}\right)\right)=\left(f_{0}, w_{k}\right)(1 \leq k \leq 18),\left(\varphi_{i}\left(f_{0}\right)^{2}\right)=\left(f_{0}^{2}\right) .
$$

Since $m=\operatorname{det} Q$, the first 18 equalities are equivalent to that $\left(c_{i k}\right)_{k=1}^{18}$ is the (minus) the $i$-th low of the adjoint matrix $\tilde{Q}$ of $Q$. Thus $c_{i k} \in \mathbf{Z}$. The last equality is equivalent to

$$
2 \gamma_{i}+\left(\left(\sum_{k=1}^{18} c_{i k} w_{k}\right)^{2}\right)=0 .
$$

Since $\Lambda$ is even, we have $\gamma_{i} \in \mathbf{Z}$. Now $\varphi_{i}$ is an isometry of $L$ for these $c_{i k}$ and $\gamma_{i}$.

q.e.d.

Lemma 7.7. Let $\varphi_{i}$ be an isometry of $L$ in Lemma (7.6). Then, for each $i(1 \leq i \leq 18)$, there is a positive integer $k_{i}$ such that the isometry $\left(\varphi_{i}^{k_{i}}, i d_{\bar{T}}\right) \in \mathrm{O}(L) \times \mathrm{O}(\bar{T})$ extends to an isometry $\Phi_{i}$ of $\Lambda$.

Proof. Let $L^{*}$ be the dual (over-) lattice of $L$. We may choose $k_{i}$ so that the induced action of $\varphi_{i}^{k_{i}}$ on $L^{*} / L$ is identity. So, we may put $k_{i}=\left|L^{*} / L\right|$.

q.e.d.

Proposition 7.8. Let $\Phi_{i}$ be an isometry of $\Lambda$ in Lemma (7.7). Then, for each $i(1 \leq i \leq 18)$, there is an automorphism $F_{i} \in \operatorname{Aut}(S)$ such that $F_{i}^{*}=\Phi_{i}$.

Proof. By the global Torelli theorem for K3 surfaces (see e.g., [BHPV]), we may show that $\Phi_{i}$ is an effective Hodge isometry of $\Lambda=H^{2}(S, \mathbf{Z})$. By the construction, $\Phi_{i}(T)=T$ and $\Phi_{i} \mid T=i d_{T}$. In particular, $\Phi_{i}\left(\sigma_{S}\right)=\sigma_{S}$. Thus $\Phi_{i}$ is a Hodge isometry of $H^{2}(S, \mathbf{Z})$. Recall that $N=N S(S)$ does not represent a (-2)-element. Thus, $S$ contains no smooth rational curve. It follows that the Kähler cone $\mathcal{K}(S)$ of $S$ coincides with the positive cone $\mathcal{C}$ of $H^{1,1}(S, \mathbf{R})$. By replacing $e_{0}$ by $-e_{0}$ (if necessary), we may assume that $e_{0}$ is in the boundary of $\mathcal{C}$. Since $\Phi_{i}\left(e_{0}\right)=e_{0}$, we have $\Phi_{i}(\mathcal{C})=\mathcal{C}$. Hence, $\Phi_{i}(\mathcal{K}(S))=\mathcal{K}(S)$ and $\Phi_{i}$ is also effective.

q.e.d.

The next proposition will complete the proof of Theorem (7.5) and hence that of Theorem (7.1):

Proposition 7.9. Let $F_{i}$ be the automorphism of $S$ in Proposition (7.8). Then:

(1) Let $G:=\left\langle F_{i}\right\rangle_{i=1}^{18}$ be a subgroup of Aut $(S)$ generated by $F_{i}$ 's. Then $G$ is a free abelian group of rank 18.

(2) Aut $(S)$ is an almost abelian group of rank 18. 
Proof. Let $g \in G$. Then, we have $g(L)=L$, and more precisely $g^{*}\left(e_{0}\right)=e_{0}, g^{*}\left(w_{i}\right)=w_{i}+m_{i}(g) w_{i}$ and $g^{*}\left(f_{0}\right)=f_{0}+\gamma(g) e_{0}+\sum c_{k}(g) w_{k}$ for some integers $m_{i}(g) \gamma(g)$ and $c_{k}(g)$. This is because the corresponding equality holds for the generators $F_{i}^{*}$ by $F_{i}^{*} \mid L=\varphi_{i}$ (see Lemma (7.6)). Then, we have a group homomorphism $\alpha: A \longrightarrow \mathbf{Z}^{18}$ defined by $g \mapsto\left(m_{i}(g)\right)_{i=1}^{18}$. Note also that $g^{*} \mid \bar{T}=i d_{\bar{T}}$, because so are $F_{i}^{*}$. Using this information, one can show that $\alpha(g)=0$ iff $g^{*} \mid \Lambda=i d$ in the same manner as in Proposition (5.1). However, $g^{*} \mid \Lambda=i d$ iff $g=i d$ by the global Torelli theorem. Thus $\alpha$ is injective. Combining this with the fact that $\alpha\left(F_{i}\right)=\left(\delta_{i k} m\right)_{k=1}^{18} \in \mathbf{Z}^{18}$, where $m$ is a positive integer in Lemma (7.6), we obtain assertion (1).

Let us show (2). We freely use the facts about almost abelian group in Section 8. By the main part of Theorem (1.5)(2), Aut $(S)$ is almost abelian of rank $r \leq 18$, i.e., there is a normal subgroup $H$ of $\operatorname{Aut~}(S)$ such that $[G: H]<\infty$ and $H$ fits in with the exact sequence

$$
1 \longrightarrow N \longrightarrow H \longrightarrow \mathbf{Z}^{r} \longrightarrow 1,
$$

where $N$ is a finite subgroup of $H$. We need to show that $r=18$. The exact sequence above induces the following exact sequence

$$
1 \longrightarrow N \cap G \longrightarrow H \cap G \longrightarrow \mathbf{Z}^{r} \text {. }
$$

Since $G$ is a free abelian group, we have $N \cap G=\{0\}$. Thus, $H \cap G$ is a subgroup of $\mathbf{Z}^{r}$. On the other hand, since $H$ is of finite index in Aut $(S)$ and $F_{i} \in \operatorname{Aut}(S)$, there is a positive integer $n$ such that

$$
F_{1}^{n}, F_{2}^{n}, \cdots, F_{18}^{n} \in H,
$$

and thus $H \cap G$ contains a subgroup isomorphic to $\mathbf{Z}^{18}$. Hence $18 \leq r$, and therefore $r=18$. q.e.d.

\section{Almost abelian group (Appendix)}

This section is a sort of appendix, in order to make statements and arguments in the main part (Sections 1-7) clear. We shall give a precise definition of the almost abelian group (that we employed) and remark on a few easy properties of almost abelian groups (that we used in the main part).

Definition 8.1. A group $G$ is an almost abelian group (resp. almost abelian of finite rank, say $r$ ) if there are a normal subgroup $G^{(0)}$ of $G$ of finite index, a finite group $K$, and an abelian group $A$ (resp. a non-negative integer $r$ ), which fit in the exact sequence

$$
1 \longrightarrow K \longrightarrow G^{(0)} \longrightarrow A \longrightarrow 0
$$

(resp.

$$
\left.1 \longrightarrow K \longrightarrow G^{(0)} \longrightarrow \mathbf{Z}^{r} \longrightarrow 0\right)
$$


It is clear that if $G$ is almost abelian, then so are subgroups $H<G$ and quotient groups $G / N$.

First, we show the well-definedness of the rank of an almost abelian group.

Lemma 8.2. Let $G$ be an almost abelian group of finite rank. Then the rank of $G$ is uniquely determined by $G$.

Proof. Let $G, G^{0}$ and $r$ be as in the definition (8.1). Let $G^{(1)}$ be another normal subgroup of $G$ of finite index which fits in an exact sequence

$$
1 \longrightarrow N \longrightarrow G^{(1)} \longrightarrow \mathbf{Z}^{s} \longrightarrow 0 .
$$

Here $N$ is a finite group. We need to show that $r=s$. Set $G^{(2)}=$ $G^{(0)} \cap G^{(1)}$. From the standard exact sequence

$$
1 \longrightarrow G^{(0)} \longrightarrow G \longrightarrow G / G^{(0)} \longrightarrow 1,
$$

we have an exact sequence

$$
1 \longrightarrow G^{(2)} \longrightarrow G^{(1)} \longrightarrow G / G^{(0)} .
$$

Thus, $G^{(2)}$ is a normal subgroup of $G^{(1)}$ of finite index. From the first exact sequence, we have an exact sequence

$$
1 \longrightarrow N \cap G^{(2)} \longrightarrow G^{(2)} \longrightarrow \mathbf{Z}^{s} .
$$

Since $N$ is a finite group and $\left[G^{(1)}: G^{(2)}\right]<\infty$, the image of the last map is also of finite index in $\mathbf{Z}^{s}$. Thus, it is isomorphic to $\mathbf{Z}^{s}$ (as abstract abelian groups) and we have an exact sequence

$$
1 \longrightarrow N^{(2)} \longrightarrow G^{(2)} \longrightarrow \mathbf{Z}^{s} \longrightarrow 0,
$$

where $N^{(2)}$ is a finite subgroup of $G^{(2)}$. Similarly, we have an exact sequence

$$
1 \longrightarrow K^{(2)} \longrightarrow G^{(2)} \longrightarrow \mathbf{Z}^{r} \longrightarrow 0,
$$

where $K^{(2)}$ is a finite subgroup of $G^{(2)}$. Taking $\operatorname{Hom}(*, \mathbf{Z})$ as groups, we obtain exact sequences

$$
\begin{aligned}
& 0 \longrightarrow \operatorname{Hom}\left(\mathbf{Z}^{r}, \mathbf{Z}\right) \longrightarrow \operatorname{Hom}\left(G^{(2)}, \mathbf{Z}\right) \longrightarrow \operatorname{Hom}\left(K^{(2)}, \mathbf{Z}\right)=0, \\
& 0 \longrightarrow \operatorname{Hom}\left(\mathbf{Z}^{s}, \mathbf{Z}\right) \longrightarrow \operatorname{Hom}\left(G^{(2)}, \mathbf{Z}\right) \longrightarrow \operatorname{Hom}\left(N^{(2)}, \mathbf{Z}\right)=0 .
\end{aligned}
$$

For the last two equalities, we used the fact that $K^{(2)}$ and $N^{(2)}$ are finite. Thus,

$$
\mathbf{Z}^{r} \simeq \operatorname{Hom}\left(G^{(2)}, \mathbf{Z}\right) \simeq \mathbf{Z}^{s} .
$$

Hence $r=s$ by the fundamental theorem of finitely generated abelian groups.

q.e.d.

The next proposition has been frequently used in the proof of Theorem (1.5):

Proposition 8.3. Let $G$ be a group. 
(1) Let

$$
1 \longrightarrow H \longrightarrow G \longrightarrow Q \longrightarrow 1
$$

be an exact sequence such that $Q$ is a finite group. Then, $H$ is an almost abelian group if and only if so is $G$. Moreover, the ranks of $H$ and $G$ are the same.

(2) Let

$$
1 \longrightarrow K \longrightarrow G \longrightarrow H \longrightarrow 1
$$

be an exact sequence such that $K$ is a finite group. Then, $H$ is an almost abelian group if and only if so is $G$. Moreover, the ranks of $H$ and $G$ are the same.

Proof. We shall show (1). The proof of (2) is similar. Assume that $G$ is almost abelian. Take a normal subgroup $G^{(0)}$ as in the definition (8.1). Set $H^{(0)}:=G^{(0)} \cap H$. Then, as in the proof of Lemma $(8.2), H^{(0)}$ makes $H$ almost abelian of the same rank as $G$. This shows "if part". Assume that $H$ is almost abelian and take a normal subgroup $H^{(0)}$ of $H$ as in the Definition (8.1). Then

$$
G^{(0)}:=\cap_{[g] \in G / H} g^{-1} H^{(0)} g
$$

is a normal subgroup of $G$ of finite index. As before, $G^{(0)}$ makes $G$ almost abelian of the same rank of $H$. This shows "only if part". q.e.d.

\section{References}

[BHPV] W. Barth, K. Hulek, C. Peters, \& A. Van de Ven, Compact Complex Surfaces, Springer-Verlag, 2004, MR 2030225, Zbl 1036.14016.

[Be1] A. Beauville, Variété Kähleriennes dont la premiére classe de chern est nulle, J. Differential Geom. 18 (1983) 755-782, MR 0730926, Zbl 0537.53056.

[Be2] Some remarks on Kähler manifolds with $c_{1}=0$, in 'Classification of algebraic and analytic manifolds' (Katata, 1982), Progr. Math. Birkhauser Boston, Boston, MA 39 (1983) 1-26, MR 0728605, Zbl 0537.53057.

[CP] F. Campana \& T. Peternell, Towards a Mori theory on compact Kähler threefolds, I, Math. Nachr. 187 (1997) 29-59, MR 1471137, Zbl 0889.32027.

[DS1] T.C. Dinh \& N. Sibony, Groupes commutatifs d'autmorphismes d'une variété Kählerienne compacte, Duke Math. J. 123 (2004) 311-328, MR 2066940, Zbl 1065.32012.

[DS2] Une borne supérieure pour l'entropie topologique d'une application rationnelle, Ann. of Math. 161 (2005) 1637-1644, MR 2180409, Zbl 1084.54013.

[DS3] Green currents for holomorphic automorphisms of compact Kähler manifolds, J. Amer. Math. Soc. 18 (2005) 291-312, MR 2137979, Zbl 1066.32024.

[Fu1] A. Fujiki, A theorem on bimeromorphic maps of Kähler manifolds and its applications, Publ. Res. Inst. Math. Sci. 17 (1981) 735-754, MR 0642659, Zbl 0515.53049. 
[Fu2] _ On primitively symplectic compact Kähler $V$-manifolds of dimension four, in 'Classification of algebraic and analytic manifolds' (Katata, 1982), Progr. Math., Birkhauser Boston 39 (1983) 71-250, MR 0728609, Zbl 0549.32018.

[Fu3] On the de Rham cohomology group of a compact Kähler symplectic manifold, in 'Algebraic geometry' (Sendai, 1985), Adv. Stud. Pure Math. 10 (1987) 105-165, MR 0946237, Zbl 0654.53065.

[GHJ] M. Gross, D. Huybrechts, \& D. Joyce, Calabi-Yau manifolds and related geometries, Springer-Verlag, 2003, MR 1963559, Zbl 1001.00028.

[GM] B.H. Gross \& C.T. McMullen, Automorphisms of even unimodular lattices and unramified Salem numbers, J. Algebra 257 (2002) 265-290, MR 1947324, Zbl 1022.11016.

[He] S. Helgason, Differential geometry, Lie groups, and symmetric spaces, Pure and Applied Mathematics, 80, Academic Press New York-London, 1978, MR 0514561, Zbl 0451.53038.

[Hi] H. Hironaka, Desingularization of complex-analytic varieties, in 'Actes du Congres International des Mathematiciens' (Nice, 1970), 627-631, GauthierVillars, Paris, 1971, MR 0425170, Zbl 0231.32007.

[HLOY] S. Hosono, B.H. Lian, K. Oguiso, \& S.-T. Yau, Kummer structures on a K3 surface: An old question of T. Shioda, Duke Math. J. 120 (2003) 577-599, MR 2030099, Zbl 1051.14046.

[Hu] D. Huybrechts, Compact hyperkähler manifolds: basic results, Invent. Math. 135 (1999) 63-113; Erratum: "Compact hyperkähler manifolds: basic results", Invent. Math. 152 (2003) 209-212, MR 1664696, MR 1965365, Zbl 0953.53031, Zbl 1029.53058.

[Kb] S. Kobayashi, Transformation groups in differential geometry, SpringerVerlag, 1972, MR 0355886, Zbl 0246.53031.

[Mc] C.T. McMullen, Dynamics on K3 surfaces: Salem numbers and Siegel disks, J. Reine Angew. Math. 545 (2002) 201-233, MR 1896103, Zbl 1054.37026.

[Ni] V.V. Nikulin, Finite groups of automorphisms of Kähler K3 surfaces, Trans. Moscow Math. Soc. 38 (1980) 71-135, MR 0544937, Zbl 0454.14017.

[Og1] K. Oguiso, Local families of $K 3$ surfaces and applications, J. Algebraic Geom. 12 (2003) 405-433, MR 1966023, Zbl 1085.14510.

[Og2] _ Automorphisms of hyperkähler manifolds in the view of topological entropy, to appear in the Proceedings of Conference on Algebraic Geometry in honour of Prof. Dolgachev, July 2004; Contemparary Math. AMS.

[Og3] , Tits alternative in hypekähler manifolds, Math. Research. Letters 13 (2006) 307-316, MR 2231119.

[Sh] T. Shioda, On the Mordell-Weil lattices, Comment. Math. Univ. St. Paul. 39 (1990) 211-240, MR 1081832, Zbl 0725.14017.

[Si] Y.T. Siu, Every K3 surface is Kähler, Invent. Math. 73 (1983) 139-150, MR 07073521, Zbl 0557.32004.

[Ta] T. Takagi, Algebraic number theory, 2nd edition, 1971, Iwamani Shoten (in Japanese).

[Vo] C. Voisin, On the homotopy types of compact Kähler and complex projective manifolds, Invent. Math. 157 (2004) 329-343, MR 2076925, Zbl 1065.32010. 
[VZ] E. Viehweg \& K. Zuo, On the isotriviality of families of projective manifolds over curves, J. Algebraic Geom. 10 (2001) 781-799, MR 1838979, Zbl 1079.14503.

[Ya1] S.-T. Yau, On the Ricci curvature of a compact Kähler manifold and the complex Monge-Ampere equation, I, Comm. Pure Appl. Math. 31 (1978) 339-411, MR 0480351, Zbl 0369.53059.

[Ya2] Calabi's conjecture and some new results in algebraic geometry, Proc. Nat. Acad. Sci. USA 74 (1977) 1798-1799, MR 0451180, Zbl 0355.32028.

KEIO UNIVERSITY Raiosha, 4-1-1 Hiyoshi Kounoku-Ku Yokohama 223-8521, JAPAN and

Korea Institute for Advanced Study 207-43 ChEONRYANGNI-2DONG Dongdaemun-Gu, SeOul 130-722

KoreA

E-mail address: oguiso@hc.cc.keio.ac.jp 